NBER WORKING PAPER SERIES

\title{
THE MACROECONOMIC ANNOUNCEMENT PREMIUM
}

\author{
Jessica A. Wachter \\ Yicheng Zhu \\ Working Paper 24432 \\ http://www.nber.org/papers/w24432 \\ NATIONAL BUREAU OF ECONOMIC RESEARCH \\ 1050 Massachusetts Avenue \\ Cambridge, MA 02138 \\ March 2018
}

We thank Frank Diebold, Winston Dou, Marco Grotteria, Nick Roussanov, Chaojun Wang, and seminar participants at Wharton for helpful comments. The views expressed herein are those of the authors and do not necessarily reflect the views of the National Bureau of Economic Research.

NBER working papers are circulated for discussion and comment purposes. They have not been peer-reviewed or been subject to the review by the NBER Board of Directors that accompanies official NBER publications.

(C) 2018 by Jessica A. Wachter and Yicheng Zhu. All rights reserved. Short sections of text, not to exceed two paragraphs, may be quoted without explicit permission provided that full credit, including (C) notice, is given to the source. 
The Macroeconomic Announcement Premium

Jessica A. Wachter and Yicheng Zhu

NBER Working Paper No. 24432

March 2018

JEL No. G11,G12

\begin{abstract}
Empirical studies demonstrate striking patterns in stock market returns in relation to scheduled macroeconomic announcements. First, a large proportion of the total equity premium is realized on days with macroeconomic announcements, despite the small number of such days. Second, the relation between market betas and expected returns is far stronger on announcement days as compared with non-announcement days. Third, risk as measured by volatilities and betas is equal on both types of days. We present a model with rare events that jointly explains these phenomena. In our model, which is solved in closed form, agents learn about a latent disaster probability from scheduled announcements. We quantitatively account for the empirical findings, along with other facts about the market portfolio.
\end{abstract}

Jessica A. Wachter

Department of Finance $2300 \mathrm{SH}-\mathrm{DH}$

The Wharton School University of Pennsylvania

3620 Locust Walk Philadelphia, PA 19104

and NBER

jwachter@wharton.upenn.edu

Yicheng Zhu

The Wharton School

Philadelphia, PA 19104

yichengz@wharton.upenn.edu 


\section{Introduction}

Since the work of Sharpe (1964) and Lintner (1965), the Capital Asset Pricing Model (CAPM) has been the benchmark model of the cross-section of asset returns. While generalizations have proliferated, the CAPM, with its simple and compelling structure and tight empirical predictions, remains the major theoretical framework for understanding the relation between risk and return. Recently, Savor and Wilson (2014) document a striking fact about the fit of the CAPM. Despite its poor performance in explaining the cross section overall, the CAPM does quite well on a subset of trading days, namely those days in which the Federal Open Market Committee (FOMC) or the Bureau of Labor Statistics (BLS) releases macroeconomic news.

Figure 1 reproduces the main result of Savor and Wilson (2014) using updated data. We sort stocks into portfolios based on market beta (the covariance with the market divided by market variance) computed using rolling windows. We display the relation between portfolio beta and expected returns on announcement days and nonannouncement days in the data. This relation is known as the security market line. On non-announcement days (the majority), the slope is indistinguishable from zero. That is, there appears to be no relation between beta and expected returns. This result holds unconditionally, and is responsible for the widely-held view of the poor performance of the CAPM. However, on announcement days, a strong positive relation between betas and expected returns appears. Moreover, portfolios line up well against the security market line, suggesting that the relation is not only strong, but that the total explanatory power is high.

These findings closely relate to a recent empirical literature (Lucca and Moench, 2015; Savor and Wilson, 2013) demonstrating that market returns are much higher on announcement days than non-announcement days. One potential explanation is that risk is different on announcement and non-announcement days. However in the data, variances and covariances on announcement days are nearly indistinguishable from those on non-announcement days. This deepens the puzzle, ruling out a number of possible explanations.

We can summarize the facts as follows:

1. The slope of the security market line is higher on announcement days than on nonannouncement days. The difference is economically and statistically significant. 
2. The security market line is essentially flat on non-announcement days.

3. The equity premium is much higher on announcement days as opposed to nonannouncement days

4. Volatilities and betas with respect to the market are the same on both types of days.

In this paper, we build a frictionless model with rational investors that explains all four findings. Our model is relatively simple and solved in closed form, allowing us to clearly elucidate the elements of the theory that are necessary to explain these results. Nonetheless, the model is quantitatively realistic, in that we explain not only these findings above, but also the overall risk and return of the aggregate stock market.

One important aspect of our model is that, despite the lack of frictions, investors do not have complete information. Macroeconomic announcements matter for stock prices because they reveal information to investors. This only makes sense if investors do not have full information in the first place 1 The information that is revealed matters greatly to investors, which is why a premium is required to hold stocks on announcement days. In our model, the information concerns the likelihood of economic disaster similar to the Great Depression or what many countries suffered following the 2008 financial crisis. Constant relative risk aversion implies that such deep downturns matter more to investors than what would be suggested purely on the basis of second moments.

We further assume that stocks have differential exposure to macroeconomic risk. We endogenously derive the exposure on stock returns from the exposure of the underlying cash flows. We also assume, plausibly, that there is some variability in the probability of disaster that is not revealed in the macroeconomic announcements. Stocks with greater exposure have endogenously higher betas, both on announcement and nonannouncement days, than those with lower exposure. They have much higher returns, in line with the data, on announcement days, because that is when a disproportionate amount of information is revealed.

We find that the presence of rare events breaks the traditional relation between

\footnotetext{
${ }^{1}$ Another possibility is that macroeconomic announcements themselves create the risk perhaps because they reflect on the competence of the Federal Reserve. We do not consider that possibility here.
} 
risk and return. This is key, because findings 1-4 together show a dramatic failure of the risk/return relation. In the model, risk appears to be the same on announcement and non-announcement days because of the asymmetric nature of the rare event. Most likely, investors will learn that the economy continues to be in good shape and the risk of disaster remains low. There is a small probability, however, that they will learn that the economy is in worse shape than believed.

While we focus on macroeconomic announcements, the tools we develop could be used to address other types of periodic information revelation. There is a vast empirical literature on announcement effects (La Porta et al., 1997; Fama, 1970), of which the literature on macro-announcements is a part. There is, at present, scant theo-

retical work (Ai and Bansal (2017) is a recent exception). In this paper, we develop a set of theoretical tools to handle the fact that announcements occur at deterministic intervals, and that a finite amount of information is released over a vanishingly small period of time. Time just before and just after the announcement is connected through intertemporal optimization conditions. We show that these conditions form a set of boundary conditions for the dynamic evolution of prices in the interval between announcements. It is this insight that allows us to solve the model in closed form.

The rest of the paper proceeds as follows. Section 2 discusses the model. Section 3 discusses the fit of the model to the data, and Section 4 concludes.

\section{A model with announcement effects}

\subsection{Endowment and preferences}

We assume an endowment economy with an infinitely-lived representative agent. Aggregate consumption (the endowment) follows the stochastic process

$$
\frac{d C_{t}}{C_{t^{-}}}=\mu_{C} d t+\sigma d B_{C t}+\left(e^{Z_{t}}-1\right) d N_{t}
$$

where $B_{C t}$ is a standard Brownian motion and where $N_{t}$ is a Poisson process. The diffusion term $\mu_{C} d t+\sigma d B_{C t}$ represents the behavior of consumption during normal times. The Poisson term $\left(e^{Z_{t}}-1\right) d N_{t}$ represents rare disasters. The random variable $Z_{t}$ represents the effect of a disaster on log consumption growth. We assume, for 
tractability, that $Z_{t}$ has a time-invariant distribution, which we call $\nu$; that is, $Z_{t}$ is iid over time, and independent of all other shocks. We use the notation $E_{\nu}$ to denote expectations taken over $\nu$.

We assume the representative agent has recursive utility with EIS equal to 1, which gives us closed-form solutions up to ordinary differential equations. We use the continuous-time characterization of Epstein and Zin (1989) derived by Duffie and Epstein (1992). The following recursion characterizes utility $V_{t}$ :

$$
V_{t}=\max E_{t} \int_{t}^{\infty} f\left(C_{s}, V_{s}\right) d s
$$

where

$$
f\left(C_{t}, V_{t}\right)=\beta(1-\gamma) V_{t}\left(\log C_{t}-\frac{1}{1-\gamma} \log \left[(1-\gamma) V_{t}\right]\right) .
$$

Here $\beta$ represents the rate of time preference, and $\gamma$ represents relative risk aversion. The case of $\gamma=1$ collapses to time-additive $(\log )$ utility. When $\gamma \neq 1$, preferences satisfy risk-sensitivity, the characteristic that Ai and Bansal (2017) show is a necessary condition for a positive announcement premium.

\subsection{Scheduled announcements and the disaster probability}

We assume that scheduled announcements convey information about the probability of a rare disaster (in what follows, we use the terminology probability and intensity interchangeably). The probability may also vary over time for exogenous reasons; this creates volatility in stock prices in periods that do not contain announcements.

To parsimoniously capture these features in the model, we assume the intensity of $N_{t}$ is a sum of two processes, $\lambda_{1 t}$ and $\lambda_{2 t} 2^{2}$ We assume investors observe $\lambda_{2 t}$, which follows

$$
d \lambda_{2 t}=-\kappa\left(\lambda_{2 t}-\bar{\lambda}_{2}\right) d t+\sigma_{\lambda} \sqrt{\lambda_{2 t}} d B_{\lambda t}
$$

with $B_{\lambda t}$ a Brownian motion independent of $B_{C t}$. The process for $\lambda_{2 t}$ is the same as the one assumed for the disaster probability in Wachter (2013).

\footnotetext{
${ }^{2}$ Equivalently, decompose, $N_{t}$ as

$$
N_{t}=N_{1 t}+N_{2 t}
$$

where $N_{j t}$, for $j=1,2$, has intensity $\lambda_{j t}$.
} 
The intensity $\lambda_{1 t}$ follows a Markov switching process. Following Benzoni et al. (2011), we assume two states, $\lambda^{G}$ (good) and $\lambda^{B}$ (bad), with $0 \leq \lambda^{G}<\lambda^{B}$, and

$$
\begin{aligned}
& P\left(\lambda_{1, t+d t}=\lambda^{G} \mid \lambda_{1 t}=\lambda^{B}\right)=\eta_{B G} d t \\
& P\left(\lambda_{1, t+d t}=\lambda^{B} \mid \lambda_{1 t}=\lambda^{G}\right)=\eta_{G B} d t .
\end{aligned}
$$

If the economy is in a good state, the probability of a switch to the bad state over the next instant is $\eta_{G B} d t>0$. If the economy is in a bad state, the probability of a switch to the good state is $\eta_{B G} d t>0$.

The intensity $\lambda_{1 t}$ is latent; investors learn about it from macroeconomic announcements. Let $p_{t}$ denote the probability that the representative agent places on $\lambda_{1 t}=\lambda^{B}$. Between announcements, we assume $p_{t}$ evolves according to

$$
d p_{t}=\left(-p_{t} \eta_{B G}+\left(1-p_{t}\right) \eta_{G B}\right) d t=\left(-p_{t}\left(\eta_{G B}+\eta_{B G}\right)+\eta_{G B}\right) d t
$$

This assumption implies that the agent learns only from announcements.$^{3}$ If the economy is in a good state, which it is with probability $1-p_{t}$, the chance of a shift to the bad state over the next instant is $\eta_{G B} d t$. If the economy is in a bad state, which is with probability $p_{t}$, the chance of a shift to the good state over the next instant is $\eta_{B G} d t$. Define

$$
\bar{\lambda}_{1}\left(p_{t}\right) \equiv p_{t} \lambda^{B}+\left(1-p_{t}\right) \lambda^{G}
$$

as the agent's posterior value of $\lambda_{1 t}$.

Announcements convey information about $\lambda_{1 t}$. For simplicity, we assume announce-

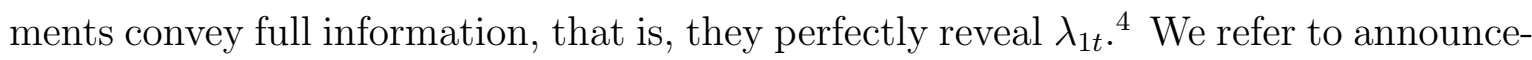

${ }^{3}$ Bayesian learning of $p_{t}$ implies

$$
d p_{t}=p_{t}\left(\frac{\lambda^{B}-\bar{\lambda}_{1}\left(p_{t}\right)}{\bar{\lambda}_{1}\left(p_{t}\right)}\right) d N_{1 t}+\left(-p\left(\lambda^{B}-\bar{\lambda}_{1}\left(p_{t}\right)\right)-p_{t} \eta_{B G}+\left(1-p_{t}\right) \eta_{G B}\right) d t
$$

(Liptser and Shiryaev, 2001). The first term multiplying $N_{1 t}$ corresponds to the actual effect of disasters. The term $-p\left(\lambda^{B}-\bar{\lambda}_{1}\left(p_{t}\right)\right)$ in the drift corresponds to the effect of no disasters. We abstract from these effects in $(7)$. Because disasters will be very unlikely, the term $-p\left(\lambda^{B}-\bar{\lambda}_{1}\left(p_{t}\right)\right)$ is small (agents do not learn much from the fact that disasters do not occur). In what follows, we compare the data to simulations that do not contain disasters. Therefore ignoring the Poisson term can be understood as an implementation of realization utility, defined by Cogley and Sargent (2008). We allow agents to learn from disasters; however, they do not forecast that they will learn from disasters.

${ }^{4}$ In effect, we assume the government body issuing the announcement has better information, perhaps because of superior access to data. Stein and Sunderam (2017) model the strategic problem of the announcer and investors, and show that announcements might reveal more information than a 
ments revealing $\lambda_{1 t}$ to be $\lambda^{G}$ as positive and those revealing it to be $\lambda^{B}$ as negative. The reason for this terminology is intuitive: an announcement revealing the disaster probability to be low should be good news. The following sections make this intuition precise.

Let $T$ be the length of time between announcements..$^{5}$ Define $\tau$ as the time elapsed since the most recent announcement:

$$
\tau \equiv t \bmod T
$$

It is useful to keep track of the content of the most recent announcement, because of the information it conveys about the evolution of the disaster probability. Let

$$
p_{0 t} \equiv p_{t-\tau}
$$

That is, $p_{0 t}$ is the revealed probability of a bad state at the most recent announcement. By definition, $p_{0 t} \in\{0,1\}$. The process for $p_{t}$ is right-continuous with left limits. In the instant just before the announcement it is governed by (6). On the announcement itself, it jumps to 0 or 1 depending on the true (latent) value of $\lambda_{1 t}$.

Furthermore, we define

$$
\begin{aligned}
\mathcal{A} & \equiv\{t: t \bmod T=0\} \\
\mathcal{N} & \equiv\{t: t \bmod T \neq 0\}
\end{aligned}
$$

That is, $\mathcal{A}$ is the set of announcement times, and $\mathcal{N}$ is the set of non-announcement times. It is straightforward to show that $\mathcal{N}$ is an open set, and for functions defined on $\mathcal{N}$, derivatives are well-defined.

Under these assumptions, $p_{t}$ has an exact solution:

Lemma 1. Between announcements, the probability assigned to the bad state satisfies $p_{t}=p\left(\tau ; p_{0 t}\right)$, where

$$
p\left(\tau ; p_{0 t}\right)=p_{0 t} e^{-\left(\eta_{B G}+\eta_{G B}\right) \tau}+\frac{\eta_{G B}}{\eta_{B G}+\eta_{G B}}\left(1-e^{-\left(\eta_{B G}+\eta_{G B}\right) \tau}\right) .
$$

naive interpretation would suggest.

${ }^{5}$ In the data, announcements are periodic, but, depending on the type of announcement, the period length is not precisely the same. Our assumption of an equal period length is a convenient simplification that has little effect on our results. 
Proof. Equation 6 implies that $p_{t}$ is deterministic between announcements. Moreover, $p_{t}$ is memoryless in that it contains no information prior to the most recent announcement. Because the information revealed at the most recent announcement is summarized in $p_{0}$, any solution for (6) takes the form $p_{t}=p\left(\tau ; p_{0 t}\right)$, where $\tau=t \bmod T$ and $p_{0 t} \in\{0,1\}$. It follows directly from (6) that $p\left(\tau ; p_{0 t}\right)$ satisfies

$$
\frac{d}{d \tau} p\left(\tau ; p_{0}\right)=-p\left(\tau ; p_{0}\right)\left(\eta_{B G}+\eta_{G B}\right)+\eta_{G B}, \quad \tau \in[0, T) .
$$

This has a general solution:

$$
p\left(\tau ; p_{0}\right)=K_{p_{0}} e^{-\left(\eta_{B G}+\eta_{G B}\right) t}+\frac{\eta_{G B}}{\eta_{B G}+\eta_{G B}},
$$

where $K_{p_{0}}$ is a constant that depends on $p_{0}$. The boundary condition

$$
p\left(0 ; p_{0}\right)=p_{0}
$$

pins down the constant $K_{p_{0}}$, implying 10$)$.

Equation 10 shows that $p_{t}$ is a weighted average of two probabilities. The first, $p_{0 t}$, is the probability of the bad state, revealed in the most recent announcement. The second, $\frac{\eta_{G B}}{\eta_{G B}+\eta_{B G}}$, is the unconditional probability of the bad state. As $\tau$, the time elapsed since the announcement, goes from 0 to 1 , the agent's weight shifts from the former of these probabilities to the latter.

Agents forecast the outcome of the announcement based on $p_{t}$. As we will see, intertemporal optimality conditions connecting the instant before the announcement to the announcement itself are crucial determinants of equilibrium. It is thus useful to define notation for $p_{t}$ just before the announcement. Let

$$
\begin{aligned}
& p^{G}=\lim _{\tau \rightarrow T} p(\tau ; 0) \\
& p^{B}=\lim _{\tau \rightarrow T} p(\tau ; 1) .
\end{aligned}
$$

Then $p^{G}$ is the probability that the agent assigns to a negative announcement just before the announcement is realized, if the previous announcement was positive. If the previous announcement was negative, then the agent assigns probability $p^{B}$. The values of $p^{G}$ and $p^{B}$, which are strictly between 0 and 1 , follow from 100 . 


\subsection{The state-price density}

We will value claims to future cash flows using the state-price density $\pi_{t}$. This object is uniquely determined by the utility function and by the process for the endowment. Heuristically, we can think of $\pi_{t}$ as the process for marginal utility.

Theorem 1. For $t \in \mathcal{N}$, the evolution of the state price density $\pi_{t}$ is characterized by

$$
\begin{aligned}
\frac{d \pi_{t}}{\pi_{t^{-}}}=-\left(r_{t}+\left(\bar{\lambda}_{1}\left(p_{t}\right)+\lambda_{2 t}\right)\right. & \left.E_{\nu}\left[e^{-\gamma Z_{t}}-1\right]\right) d t \\
& \quad-\gamma \sigma d B_{C t}+(1-\gamma) b_{\lambda} \sigma_{\lambda} \sqrt{\lambda_{2 t}} d B_{\lambda t}+\left[e^{-\gamma Z_{t}}-1\right] d N_{t}
\end{aligned}
$$

where $r_{t}$ is the riskless interest rate, and where

$$
b_{\lambda}=\frac{1}{(1-\gamma) \sigma_{\lambda}^{2}}\left(\beta+\kappa-\sqrt{(\beta+\kappa)^{2}-2 \sigma_{\lambda}^{2}\left[E_{\nu} e^{(1-\gamma) Z_{t}}-1\right]}\right) .
$$

Proof. See Appendix A.

The instantaneous mean growth rate of the state-price density is (as usual) the riskfree rate $r_{t}$ (to be characterized below). The state-price density jumps upward in the case of a disaster, corresponding to the effect of a large decline in consumption on marginal utility. The state-price density also changes due to normal-time changes in consumption (this term will be small), and because of changes in the disaster probability not associated with announcements $(1-\gamma) b_{\lambda} \sigma_{\lambda} \sqrt{\lambda_{2 t}} d B_{\lambda t}$. When $\gamma>1,(1-\gamma) b_{\lambda}$ is positive and so marginal utility rises when the disaster probability rises. When $\gamma<1$, marginal utility falls. ${ }^{6}$

Comparing Theorem 1 to analogous results in prior studies (see Tsai and Wachter (2015) for a survey), shows that there is no special role for announcements in the dynamics of the state-price density outside of announcement periods. Announcements enter only indirectly, through the posterior probability of the Poisson shock $d N_{t}$, through the compensation in the drift, and in the riskfree rate, given in the theorem below. This is intuitive, given that announcements occur at pre-determined intervals. The announcement cycle does affect the level of the value function, but, because it is deterministic, it does not affect marginal utilities along the optimal consumption path.

\footnotetext{
${ }^{6}$ In a more general model, whether marginal utility falls or rises depends on $\gamma$ relative to the inverse of the elasticity of intertemporal substitution. See Tsai and Wachter (2017).
} 
Announcements also affect the riskfree rate indirectly through the agent's posterior probability $\bar{\lambda}_{1}\left(p_{t}\right)$. The higher this posterior probability, the lower the riskfree rate, due both to precautionary savings and a lower expected growth rate. However, there is no direct effect of announcements $\square$

Theorem 2. The riskfree rate $r_{t}$ is given by

$$
r_{t}=\beta+\mu_{C}-\gamma \sigma^{2}+\left(\bar{\lambda}_{1}\left(p_{t}\right)+\lambda_{2 t}\right) E_{\nu}\left[e^{-\gamma Z_{t}}\left(e^{Z_{t}}-1\right)\right]
$$

Proof. See Appendix A.

Announcements, however, do have a direct effect on state-price density, on the day of the announcement itself. The following theorem characterizes the change in the state-price density due to announcements.

Theorem 3 (Announcement SDF). For $t \in \mathcal{A}$, with probability 1,

$$
\frac{\pi_{t}}{\pi_{t^{-}}}=\left(\frac{\exp \left\{\zeta_{p_{0 t}}+b_{p} p_{t}\right\}}{\exp \left\{e^{\beta T} \zeta_{p_{0 t^{-}}}+b_{p} p_{t^{-}}\right\}}\right)^{1-\gamma}
$$

where

$$
b_{p}=\frac{\left(\lambda^{B}-\lambda^{G}\right) E_{\nu}\left[e^{(1-\gamma) Z_{t}}-1\right]}{(1-\gamma)\left(\beta+\eta_{G B}+\eta_{B G}\right)},
$$

and where $\zeta_{p_{0} t}, \zeta_{p_{0 t^{-}}} \in\left\{\zeta_{0}, \zeta_{1}\right\}$ with

$$
\begin{aligned}
& e^{(1-\gamma)\left(\zeta_{0} e^{\beta T}+b_{p} p^{G}\right)}=p^{G} e^{(1-\gamma)\left(\zeta_{1}+b_{p}\right)}+\left(1-p^{G}\right) e^{(1-\gamma) \zeta_{0}} \\
& e^{(1-\gamma)\left(\zeta_{1} e^{\beta T}+b_{p} p^{B}\right)}=p^{B} e^{(1-\gamma)\left(\zeta_{1}+b_{p}\right)}+\left(1-p^{B}\right) e^{(1-\gamma) \zeta_{0}}
\end{aligned}
$$

Proof. See Appendix A.

The difference ratio of state-price densities just prior to and just after an announcement in (16) will play an important role in what follows. This ratio can be thought of as an announcement stochastic discount factor (SDF), and it will determine the risk premium for macroeconomic announcements.

${ }^{7}$ While our model implies that the riskfree rate looks no different on announcement and nonannouncement days, bonds of finite maturity would go up in price on announcement days, consistent with the empirical results of Savor and Wilson (2013). 
Equation 16 shows that this stochastic discount factor is a function of $p_{0 t}$ just before and just after the announcement and $p_{t}$ just before and just after the announcement $8^{8}$ Note that $p_{0 t} \in\{0,1\}$ and $p_{t}$ just prior to an announcement is in $\left\{p^{G}, p^{B}\right\}$. It follows (see Appendix A for details) that (18) is simply the condition that over an infinitesimal interval, the expectation of the SDF must equal 1.

Equation 16 holds "only" with probability 1. That is, there is a theoretical possibility that a disaster could coincide with an announcement. Because announcements are a set of measure zero the probability that a disaster and announcement coincide is zero, and so we can ignore the theoretical possibility when calculating expectations.

The stochastic discount factor has two components, one corresponding to a change in the announcement content $\left(\zeta_{p_{0 t}}\right)$ and the other corresponding to the posterior probability of a disaster. That the posterior probability should affect the SDF is intuitive. It follows from $\lambda^{B}>\lambda^{G}$ that $b_{p}<0$. Thus for $\gamma>1$, an increase in the posterior probability of being in a bad state increases marginal utility. Moreover, the increase in marginal utility is higher, the greater is the persistence of the probability (namely, the lower $\eta_{G B}+\eta_{B G}$ ), and the lower the discount factor $\beta$. In the numerator of this term is the instantaneous effect of a disaster on utility, multiplied by the incremental probability of disaster from being in a bad state.

However, the change in the state-price density is not only due to the change in the posterior probability. There is also an effect of the announcement itself. On the announcement, the state variable $p_{0 t}$, representing the posterior on the most recent announcement, also jumps. Recall that this variable can either be 0 or 1 , because the announcement perfectly reveals the state. The effect is thus characterized by a binary variable $\zeta_{p_{0 t}}$, whose two values satisfy the system (18). When the agent receives news about $\lambda_{1 t}$ on the announcement, she changes her $p_{t}$, and incorporates the future predictable changes in $p_{t}$ into the SDF (this is why mean reversion enters in Equation 17). The agent also incorporates forecasts of future announcements through (18).

To summarize, though the announcement is instantaneous, Theorem 3 shows that a finite amount of news is revealed, namely that $\pi_{t}$ undergoes a discrete change. This is what will produce a macroeconomic announcement premium in our model.

Given the interpretation of (18) as the announcement SDF, we would expect the

\footnotetext{
${ }^{8}$ Of course, $p_{t}$ is itself a function of $p_{0 t}$ and the time since the last announcement.
} 
change in the SDF to reflect our intuition about agent's marginal utilities. In fact it does, as the next theorem shows. The following technical result is helpful.

Lemma 2. Let $\zeta_{0}, \zeta_{1}$, and $b_{p}$ be defined as in Theorem 3. Then $b_{p}<0$ and

$$
\zeta_{0}>\zeta_{1}+b_{p}
$$

Proof. See Appendix A.

Corollary 1. For $\gamma>1$, the state-price density falls when the announcement is positive and rises when the announcement is negative.

For $\gamma<1$, the state-price density falls when the announcement is negative and rises when it is positive.

Proof. It follows from Lemma 1 that $p^{G}, p^{B} \in(0,1)$. Then $e^{(1-\gamma)\left(\zeta_{0} e^{\beta T}+b_{p} p^{G}\right)}$ in 18$)$ is a weighted average of two terms, $e^{(1-\gamma)\left(\zeta_{1}+b_{p}\right)}$ and $e^{(1-\gamma) \zeta_{0}}$ with weights strictly between 0 and 1. Similarly, $e^{(1-\gamma)\left(\zeta_{1} e^{\beta T}+b_{p} p^{B}\right)}$ in 18 is a weighted average of the same two terms, $e^{(1-\gamma)\left(\zeta_{1}+b_{p}\right)}$ and $e^{(1-\gamma) \zeta_{0}}$, again with weights strictly between 0 and 1 .

Assume that $\gamma>1$. It follows from Lemma 2 that

$$
e^{(1-\gamma)\left(\zeta_{1}+b_{p}\right)}>e^{(1-\gamma) \zeta_{0}}
$$

Thus, applying (18), we find

$$
e^{(1-\gamma)\left(\zeta_{0} e^{\beta T}+b_{p} p^{G}\right)}>e^{(1-\gamma) \zeta_{0}},
$$

and thus

$$
\left(\frac{e^{\zeta_{0}}}{e^{\zeta_{0} e^{\beta T}+b_{p} p^{G}}}\right)^{1-\gamma}<1
$$

The left hand side is $\pi_{t} / \pi_{t^{-}}$if the announcement is positive and the previous announcement was also positive. It is also true that

$$
e^{(1-\gamma)\left(\zeta_{1} e^{\beta T}+b_{p} p^{B}\right)}>e^{(1-\gamma) \zeta_{0}} .
$$

Thus

$$
\left(\frac{e^{\zeta_{0}}}{e^{\zeta_{1} e^{\beta T}+b_{p} p^{B}}}\right)^{1-\gamma}<1
$$


The left hand side is $\pi_{t} / \pi_{t^{-}}$if the announcement is positive and the previous announcement was negative. Regardless of what went before, the state-price density falls when the announcement is positive.

We use the same method to show that, when the announcement is negative, the state-price density rises. It follows from $(18)$ and Lemma 2 that

$$
e^{(1-\gamma)\left(\zeta_{0} e^{\beta T}+b_{p} p^{G}\right)}<e^{(1-\gamma)\left(\zeta_{1}+b_{p}\right)}
$$

so that

$$
\left(\frac{e^{\zeta_{1}+b_{p}}}{e^{\zeta_{0} e^{\beta T}+b_{p} p^{G}}}\right)^{1-\gamma}>1
$$

The left hand side is $\pi_{t} / \pi_{t^{-}}$when the announcement is negative and the previous announcement was positive. It also follows from (18) and Lemma 2 that

$$
e^{(1-\gamma)\left(\zeta_{1} e^{\beta T}+b_{p} p^{B}\right)}<e^{(1-\gamma)\left(\zeta_{1}+b_{p}\right)}
$$

so that

$$
\left(\frac{e^{\zeta_{1}+b_{p}}}{e^{\zeta_{1} e^{\beta T}+b_{p} p^{B}}}\right)^{1-\gamma}>1
$$

Thus, regardless of what went before, the state-price density rises when the announcement is negative. The proof for $\gamma<1$ follows similarly.

\subsection{Equity prices}

We consider a cross-section of dividend claims which differ in their sensitivity to disasters. For parsimony, we assume the claims are identical in all other respects. Let $D_{t}^{j}$ equal the time- $t$ dividend of claim $j$, for $j=1, \ldots, J$. Assume

$$
\frac{d D_{t}^{j}}{D_{t^{-}}^{j}}=\mu_{D} d t+\sigma d B_{C t}+\left(e^{\phi_{j} Z_{t}}-1\right) d N_{t} .
$$

The parameter $\phi_{j}$ determines the sensitivity of the claim to disasters. If we let $F_{t}^{j}$ denote the price of such a claim, no-arbitrage implies

$$
F_{t}^{j}=E_{t} \int_{t}^{\infty} \frac{\pi_{s}}{\pi_{t}} D_{s}^{j} d s
$$


Our model implies an analytical expression for 21 that, not surprisingly given the form of (21), takes the form of an integral over $s$. The expressions in this integral are equity strips, namely claims to a dividend payment at a single point in time $9^{9}$ To simplify the problem, we first give an analytical solution for these equity strips. We use superscript $j$ to denote quantities that depend on $\phi_{j}$ and thus are asset specific.

Theorem 4. Consider a claim to a dividend $D_{s+t}$, where the process for $D_{t}$ solves (20). Let $H^{j}\left(D_{t}, p_{t}, \lambda_{2 t}, \tau, s ; p_{0 t}\right)$ denote the time-t price of this claim. That is,

$$
H^{j}\left(D_{t}^{j}, p_{t}, \lambda_{2 t}, \tau ; p_{0 t}\right)=E_{t}\left[\frac{\pi_{t+s}}{\pi_{t}} D_{t+s}^{j}\right]
$$

Then

$$
H^{j}\left(D_{t}, p_{t}, \lambda_{2 t}, \tau, s ; p_{0 t}\right)=D_{t} \exp \left\{a_{\phi}^{j}\left(\tau, s ; p_{0 t}\right)+b_{\phi p}^{j}(s) p_{t}+b_{\phi \lambda}^{j}(s) \lambda_{2 t}\right\}
$$

where

$$
b_{\phi p}^{j}(s)=\frac{\left(\lambda^{B}-\lambda^{G}\right) E_{\nu}\left[e^{\left(\phi_{j}-\gamma\right) Z_{t}}-e^{(1-\gamma) Z_{t}}\right]}{\eta_{B G}+\eta_{G B}}\left(1-e^{-\left(\eta_{B G}+\eta_{G B}\right) s}\right), \quad s \geq 0,
$$

where $b_{\phi \lambda}^{j}(s)$ solves

$$
\frac{d b_{\phi \lambda}^{j}(s)}{d s}=\frac{1}{2} \sigma_{\lambda}^{2} b_{\phi \lambda}^{j}(s)^{2}+\left((1-\gamma) b_{\lambda} \sigma_{\lambda}^{2}-\kappa\right) b_{\phi \lambda}^{j}(s)+E_{\nu}\left[e^{\left(\phi_{j}-\gamma\right) Z_{t}}-e^{(1-\gamma) Z_{t}}\right],
$$

with boundary condition $b_{\phi \lambda}^{j}(0)=0$. Define the function $a_{\phi}^{j}$ such that

$$
\begin{aligned}
a_{\phi}^{j}\left(\tau, s ; p_{0 t}\right)= & h^{j}\left(\tau+s ; p_{0 t}\right)+ \\
& \int_{0}^{s}\left(-\beta-\mu_{C}+\mu_{D}+\lambda^{G} E_{\nu}\left[e^{\left(\phi_{j}-\gamma\right) Z_{t}}-e^{(1-\gamma) Z_{t}}\right]+\kappa \bar{\lambda}_{2} b_{\phi \lambda}^{j}(u)\right) d u
\end{aligned}
$$

for $\tau \in[0, T), s \geq 0, p_{0 t} \in\{0,1\}$. The function $h^{j}$ uniquely solves

$$
e^{h^{j}\left(u ; p_{0 t^{-}}\right)+b_{\phi p}^{j}(u-T) p_{t^{-}}}=E_{t^{-}}\left[\frac{e^{(1-\gamma)\left(\zeta_{p_{0 t}}+b_{p} p_{t}\right)}}{e^{(1-\gamma)\left(e^{\beta T} \zeta_{p_{0 t^{-}}}+b_{p} p_{t^{-}}\right)}} e^{h^{j}\left(u-T ; p_{0 t}\right)+b_{\phi p}^{j}(u-T) p_{t}}\right],
$$

for $u \geq T$ and $h^{j}(u ; \cdot)=0$ for $u \in[0, T)$.

\footnotetext{
${ }^{9}$ See Lettau and Wachter (2007).
} 
Proof. See Appendix B.

The price of a claim to a future dividend depends on the current dividend, the values of the state variables, the time since the last announcement, and the maturity $s: 10$ Note that $b_{\phi p}^{j}(s)<0$ as long as $\phi_{j}>1$, implying that an increase in the probability of a bad state implies a decrease in the price of the asset. This intuitive result reflects a tradeoff between a riskfree rate effect on the one hand and a risk premium and cash flow effect on the other. An increase in $p_{t}$ lowers the riskfree rate as shown in (15). This effect alone increases the prices of all future claims. However, there is an opposing effect due to the increased risk premium, and lower expected growth rate. When $\phi_{j}>1$, the opposing effect dominates 11 The magnitude of the response depends on the maturity of the equity strip: the longer the maturity, the greater the response. As the maturity increases to infinity, the response asymptotes to $\frac{\left(\lambda^{B}-\lambda^{G}\right) E_{\nu}\left[e^{\left(\phi_{j}-\gamma\right) Z_{t}}-e^{(1-\gamma) Z_{t}}\right]}{\eta_{B G}+\eta_{G B}}$, where $E_{\nu}\left[e^{\left(\phi_{j}-\gamma\right) Z_{t}}-e^{(1-\gamma) Z_{t}}\right]$ is the response of short-term equity (per unit of maturity) for a disaster, and $\lambda^{B}-\lambda^{G}$ is the difference in the disaster probability. Finally, $\eta_{B G}+\eta_{G B}$ reflects the persistence of the disaster probability. ${ }^{12}$

The direct effect of announcements on prices is captured in the term $h^{j}\left(\tau+s ; p_{0 t}\right)$. For very short-term equity maturing before the next announcement, announcements are not relevant and $h^{j}=0$. With this as boundary condition (27), iteratively determines the value of $h^{j}$ when an announcement lies between time $t$ and maturity at $t+s$. The recursion is, in effect, on the number of remaining announcements. Equation 27 is the Euler equation at the instant of the announcement (note that inside the expectation is the announcement SDF from Theorem 3).

The value of the firm incorporates forecasts of future $p_{t}$. Like marginal utility (Theorem 3), this effect appears in two different terms, capturing both the linear evolution and the potential for large nonlinear changes. The first term $b_{\phi p}^{j}(s)$, captures the fact that, following a jump in $p_{t}$ on the announcement, $p_{t}$ mean-reverts following a linear process. The second term, captured by the function $h^{j}$ reflects the change in forecasts of the content of future announcements.

\footnotetext{
${ }^{10}$ Note that it is not necessary to use a superscript for $D_{t}$ in 23 as this formula is valid for any $D_{t}>0$.

${ }^{11}$ Note that the EIS is equal to unity. In a model with time-additive utility, the requirement is that $\phi_{j}>\gamma$, relative risk aversion, which is much stronger. For non-unitary EIS and recursive utility, the result is more complicated. See Tsai and Wachter (2017).

${ }^{12}$ The term $b_{\phi \lambda}^{j}(s)$ is the same as that in Wachter (2013); see that paper for discussion and the closed-form solution.
} 
Corollary 2 shows that the price of the continuous stream of dividends is equal to an integral of the prices of equity strips.

Corollary 2. Let $F_{t}^{j}$ be the time-t price of an asset paying the dividend process (20) with leverage parameter $\phi_{j}$. Then

$$
F^{j}\left(D_{t}, p_{t}, \lambda_{2 t}, \tau ; p_{0 t}\right)=\int_{0}^{\infty} H^{j}\left(D_{t}, p_{t}, \lambda_{2 t}, \tau, s ; p_{0 t}\right) d s
$$

where $H^{j}$ is given by (23).

Proof. The result follows directly from Theorem 4 and the no-arbitrage condition (21).

Using the characterization of the equity price in Theorem 2 , we can sign the response to the announcement.

Corollary 3. Assume that $\phi_{j}>1$. Then $F^{j}\left(D_{t}, p_{t}, \lambda_{2 t}, \tau ; p_{0 t}\right)$ increases when the announcement is positive and decreases when the announcement is negative.

Proof. By (28), it suffices to show the result for equity strips $H^{j}$. This is shown in Appendix B.

\subsection{Risk premia}

We first consider the risk premium outside of announcement periods. Let $r_{t}^{j}$ denote the expected return on asset $j$ per unit $d t$ of time $\left(r_{t}^{j}\right.$ is defined formally in Appendix B). For $t$ such that $t \bmod T \neq 0$, the instantaneous risk premium is given in the following theorem:

Theorem 5. Consider $t$ such that $t$ mod $T \neq 0$. Then the instantaneous risk premium for an equity asset defined in section 2.4 is given by

$$
r_{t}^{j}-r_{t}=\gamma \sigma^{2}-\lambda_{2 t}(1-\gamma) b_{\lambda} \frac{1}{F_{t}^{j}} \frac{\partial F_{t}^{j}}{\partial \lambda_{2 t}} \sigma_{\lambda}^{2}-\left(\bar{\lambda}_{1}\left(p_{t}\right)+\lambda_{2 t}\right) E_{\nu}\left[\left(e^{-\gamma Z_{t}}-1\right)\left(e^{\phi_{j} Z_{t}}-1\right)\right] .
$$

The theorem divides the premium into three components: the first is the standard consumption CAPM term (negligible in our calibration). The second term is the premium investors require for baring the risk of facing risk in $\lambda_{2 t}$. Provided that the price 
falls when $\lambda_{2 t}$ rises, this is positive for $\gamma>1$. See the discussion following Theorem 1 for further detail. The third term is the premium directly linked to the rare disasters. Note that the probability of the disaster outcome is the agent's posterior probability, $\bar{\lambda}_{1}\left(p_{t}\right)+\lambda_{2 t}$. The disaster premium is positive provided that agents are risk averse and that asset has positive exposure to disasters $\phi_{j}>0$.

We now consider the risk premium on announcement dates. On non-announcement dates, the risk premium earned on the asset is equal to $\left(r_{t}^{j}-r_{t}\right) d t$. Therefore the usual continuous-time result holds: the risk premium approaches zero for sufficiently small time periods. This is not true for announcements dates.

Intuitively, the announcement premium should be given by the covariance of returns with the stochastic discount factor. The following theorem makes this precise.

Theorem 6. For assets defined in Theorem 2, the announcement premium is given by

$$
E_{t^{-}}\left[\frac{F_{t}^{j}-F_{t^{-}}^{j}}{F_{t^{-}}^{j}}\right]=-E_{t^{-}}\left[\left(\frac{\pi_{t}-\pi_{t^{-}}}{\pi_{t^{-}}}\right)\left(\frac{F_{t}^{j}-F_{t^{-}}^{j}}{F_{t^{-}}^{j}}\right)\right]
$$

for $t \bmod T=0$.

Proof. Algebra implies that

$$
E_{t^{-}}\left[\left(\frac{\pi_{t}-\pi_{t^{-}}}{\pi_{t^{-}}}\right)\left(\frac{F_{t}^{j}-F_{t^{-}}^{j}}{F_{t^{-}}^{j}}\right)\right]=E_{t^{-}}\left[\frac{\pi_{t}}{\pi_{t^{-}}} \frac{F_{t}^{j}}{F_{t^{-}}^{j}}-\frac{F_{t}^{j}}{F_{t^{-}}^{j}}-\left(\frac{\pi_{t}}{\pi_{t^{-}}}-1\right)\right]
$$

By the characterization of $F^{j}$ in 21 ,

$$
\pi_{t^{-}} F_{t^{-}}^{j}=E_{t^{-}}\left[\pi_{t} F_{t}^{j}\right]
$$

Furthermore,

$$
\pi_{t^{-}}=E_{t^{-}}\left[\pi_{t}\right]
$$

(see Appendix A). The result follows.

Corollary 4. Consider an asset with leverage parameter $\phi>1$. The announcement premium is positive if $\gamma>1$ and negative if $\gamma<1$.

Proof. Corollaries 1 and 3 show that changes in $F$ and in $\pi$ upon announcements have opposite signs when $\gamma>1$ and the same sign when $\gamma<1$. The result follows. 


\section{Quantitative results}

We start by replicating the evidence of Savor and Wilson (2014) in an extended sample. Section 3.1 describes the data and Section 3.2 the empirical findings. We then simulate repeated samples from the model described in the previous section. Section 3.3 describes the calibration of our model and Section 3.4, the simulation results. Section 3.5 discusses the model's mechanism.

\subsection{Data}

We obtain daily stock returns from the Center for Research in Security Prices (CRSP) for individual stocks traded on NYSE, AMEX, NASDAQ and ARCA from January 1961 to September 2016. In addition, we also use the daily market excess returns and risk-free rate provided by Kenneth French. The scheduled announcement dates before 2010 are provided by Savor and Wilson (2014). Following their approach, we add target-rate announcements of the FOMC and inflation and employment announcements of the BLS for the remaining dates.

We define the daily excess return to be the daily (level) return of a stock in excess of the daily return on the 1-month Treasury bill. We estimate covariances on individual stock returns with the market return using daily data and 12-month rolling windows. We include stocks which are available for trading on $90 \%$ or more of the trading days. At the start of each trading month, we sort stocks by estimated betas, and create deciles. We then form value-weighted portfolios of the stocks in each deciles, and compute daily excess returns.

\subsection{Empirical findings}

Table 1 presents summary statistics on the ten beta-sorted portfolios. For each portfolio $j, j=1, \ldots, 10$, we use the notation $E\left[R X^{j}\right]$ to denote the mean excess return, $\sigma^{j}$ the volatility of the excess return, and $\beta^{j}$ the covariance with the value-weighted market portfolio divided by the variance of the market portfolio. Table 1 shows statistics for daily returns computed over the full sample, over announcement days, and over non- 
announcement days. ${ }^{13}$ There is a weak positive relation between full-sample returns and market betas. On non-announcement days, there is virtually no relation between betas and expected returns. However, on announcement days, there is a strong relation between beta and expected returns.

Comparing results for the two types of days in Table 1 also shows that average returns on announcement days are much higher than on non-announcement days, often by a full order of magnitude. Yet the standard deviation and the betas computed over announcement days and non-announcement days are almost exactly the same.

Figure 1 shows average daily excess returns in each of the ten portfolios, plotted against the betas on the portfolios for announcement days (diamonds) and nonannouncement days (squares). Also shown is the fitted line on both days. This relation, known as the security market line, is strongly upward-sloping on announcement days, but virtually flat on non-announcement days.

\subsection{Calibration}

We now describe the calibration of the model in Section 2, We choose preference parameters and normal-times consumption parameters to be the same as in Wachter (2013). We also choose the same values for the mean reversion of the $\lambda_{2}$-process $(\kappa)$ and the volatility parameter of this process, $\sigma_{\lambda}$. For simplicity, we assume that, when the economy is in the good state, the intensity $\lambda_{1 t}$ is zero, that is $\lambda_{G}=0$. We choose $\phi_{G B}$ so that the bad state of the economy is a rare event, and $\phi_{B G}$ so that it is persistent. The unconditional probability of the bad state in our calibration is $\phi_{G B} /\left(\phi_{G B}+\phi_{B G}\right)=13 \%$. We then choose $\bar{\lambda}_{2}$ and $\lambda_{B}$ with the restriction that the average disaster probability is $3.6 \%$, as in Barro and Ursúa (2008). The values $\bar{\lambda}_{2}=2.8 \%$ and $\lambda_{B}=5.4 \%$ satisfy that restriction. The disaster distribution is taken to be multinomial, as measured in the data by Barro and Ursua. See Wachter (2013) for further detail.

We choose the disaster sensitivity $\phi_{j}$ to be uniformly distributed between 1.5 and 7. For simplicity, we assume that during normal times firm dividends grow at the same rate as each other and at the same rate as consumption $\mu_{D}=\mu_{C}$. Table 2 reports

\footnotetext{
${ }^{13}$ Betas and volatilities are computed in the standard way, as central second moments. An announcement-day volatility therefore is computed as the mean squared difference between the announcement return and the mean announcement return. Announcement-day betas are computed analogously.
} 
parameter choices.

\subsection{Simulation}

To evaluate the fit of the model, we simulate 500 artificial histories, each of length 50 years $(240 \times 50$ days $)$. We assume that announcements occur every 10 trading days. For each history, we simulate a burn-in period, so that we start the history from a draw from the stationary distribution of the state variables. We simulate the model using the true (as opposed to the investor's) distribution. When we report statistics, we consider sample paths where the economy remains in a good state $\left(\lambda_{1 t}=\lambda^{G}\right)$ and where there are no disasters. This is reasonable given that both the bad state of the economy, and a disaster, are rare events. By taking a stand on the type of events that have been observed over the short sample available to us, we considerably narrow the standard errors for the model 14

While time is continuous in our analytical model, it is necessarily discrete in our simulations. We simulate the model at a daily frequency to match the frequency of the data. We compute end-of-day prices, and assume the announcement occurs in the middle of a trading day.

Given a series of state variables and of shocks, we compute returns as follows. For each asset $j$, define the price-dividend ratio $G_{t}^{j}=F_{t}^{j} / D_{t}^{j}$. From (28), it follows that $G_{t}^{j}$ is a function of the state variables alone. We approximate the daily return as

$$
\begin{aligned}
R_{t, t+\Delta t}^{j} & \approx \frac{F_{t+\Delta t}^{j}+D_{t+\Delta t}^{j} \Delta t}{F_{t}^{j}} \\
& =\frac{D_{t+\Delta t}^{j} G_{t+\Delta t}^{j}+D_{t+\Delta t}^{j} \Delta t}{D_{t}^{j} G_{t}^{j}} \\
& =\frac{D_{t+\Delta t}^{j}}{D_{t}^{j}} \frac{G_{t+\Delta t}^{j}+\Delta t}{G_{t}^{j}} \\
& \approx \exp \left\{\bar{\mu}_{D} \Delta t-\frac{1}{2} \sigma^{2} \Delta t+\sigma\left(B_{C, t+\Delta t}-B_{C, t}\right)\right\} \frac{G_{t+\Delta t}^{j}+\Delta t}{G_{t}^{j}}
\end{aligned}
$$

where $\Delta t=1 / 240$. The last line follows because we consider sample paths with no

\footnotetext{
${ }^{14}$ Academic research also contains an element of bias in that, had we a truly unbiased sample, we might not think of the resulting effect as an anomaly.
} 
disasters. The risk free rate is approximated by

$$
R_{f t}=\exp \left(r_{t} \Delta t\right) .
$$

The daily excess return of asset $k$ is then

$$
R X_{t, t+\Delta t}^{j}=R_{t, t+\Delta t}^{j}-R_{f t} .
$$

We define the value-weighted market return just as in the data, namely we take a value-weighted portfolio of returns. We assume that the assets have the same value at the beginning of the sample. Because the assets all have the same loading on the Brownian shock and the same drift, and conditional on a history not containing rare events, the model implies a stationary distribution of portfolio weights. Given a time series of excess returns on firms (which, because we have no idiosyncratic risk, we take as analogous to portfolios), and a time series of excess returns on the market, we compute statistics exactly as in the data.

Figure 2 displays our main result. We overlay the simulated statistics on the empirical statistics from Figure 1. Each dot on the figure represents a statistic for one firm, for one simulated sample. Blue dots show pairs of average excess returns and betas on announcement days, while grey dots show pairs on non-announcement days. There is a clear separation between the two types of days, with the announcement-day statistics lining up on the announcement security market line in the data. The nonannouncement-day statistics coincide with those statistics on the non-announcement days in the data. That is, the model captures the qualitative and quantitative effect of announcement days on the security market line.

Figure 3 further clarifies the relation between the announcement and non-announcement days in the model by showing interquartile ranges. There is a clear separation between announcement and non-announcement days, and essentially a zero probability, according to the model, that the differences in security market lines could arise by chance.

A natural explanation for why risk premia are greater on announcements versus non-announcement days is that the risk is different. Table 1 shows, strikingly, that this is not true in the data. Betas computed on announcement days are virtually identical to those on non-announcement days. To be consistent with the data, a model must necessarily not only explain the difference in expected returns, but also the difference 
in betas.

The mechanism that we propose meets this requirement. Table 3 reports betas in the model from the two types of days. As in the data, they are indistinguishable. This may be surprising given that risk premia are clearly higher on announcement days. The reason, which we describe in more detail in the discussion section that follows, is that rare events imply that the beta is not a good proxy for the true risk of the portfolio. In most samples, one will observe very little extra variation on announcement days, because the news confirms expectations. Investors factor in the tail event of a negative announcement, but it need not occur.

Table 4 shows the regression slope in the data on announcement and non-announcement days, the median values from simulations in the model, and the $90 \%$ confidence intervals. We run the regression

$$
\hat{E}\left[R X_{t}^{j} \mid t \in i\right]=\delta_{i} \beta_{i}^{j}+\text { error }
$$

where $i=a$ (announcement days) or $n$ (non-announcement days). These slopes can be understood as measures of the daily market risk premia on the two types of days. In the data the slope is 10 basis points on announcements, and only 1 basis point on nonannouncement days. The model implies nearly identical results, with tight confidence intervals.

In addition, we also compute the summary statistics of the market portfolio, and the results are shown in Table 5. The important feature here is that the volatility of the market portfolio is the same on two different type of days, while the mean excess returns appear to be quite different. This table shows that the model can capture the first and second moment of the aggregate market on the two types of days, as well as of the cross-section.

\subsection{Discussion}

The previous subsection demonstrates that the model accounts for the three main findings described in the introduction. How is it that the model can account for these findings?

In the model, announcements convey important news about the distribution of 
future outcomes in the economy. On that day, it is possible that a bad state of the economy could be revealed. If the bad state is realized, not only will asset values be affected, but the marginal utility of economic agents will rise. Thus investors require a premium to hold assets over the risky announcement period.

In our model, some assets have cash flows that are more sensitive to others. The sensitivity parameter $\phi_{j}$, while not the same as the beta, is closely related. Assets with high $\phi_{j}$ have a greater dividend response to disasters. Their prices thus move more with changes in the disaster probability, and in particular with $\lambda_{1 t}$ and $\lambda_{2 t}$. The value-weighted market portfolio of course also moves with the disaster probability, and thus the higher is $\phi_{j}$ (over the relevant range), the higher is the return beta with the market, both on non-announcement days (which reveal information about $\lambda_{2 t}$, and on announcement days, which reveal additional information about $\lambda_{1 t}$.

Thus the model predicts a relation between risk and return on both announcement and non-announcement days, but because the risk is so much greater on announcement days, the premium, and therefore the spread in expected returns between low and highsensitivity assets, will also be much greater.

While this reasoning explains why the model accounts for facts $1-3$ in the introduction, it does not explain how the model accounts for fact 4. That is, one might expect that the volatility would be greater on announcement days than on non-announcement days. After all, more information is revealed on those days. Moreover, one would expect higher betas on those days. In the data, volatilities and betas are quite clearly the same on both types of days. It is surprising that it is also a property of the model.

The model breaks the link between risk and return through rare events. The lack of volatility in the model is a Peso problem: namely, the economy has been in a good state of the world, and thus positive announcements have been observed. The true population volatility on announcement days is indeed higher than on non-announcement days, but we do not observe this true population volatility. Another way to phrase this is that, if we assume no rare events, true volatility is easy to measure and is tightly captured by the second central moment over short samples. If there are rare events, true volatility is difficult to measure.

As this discussion suggests, there is also a Peso problem in the observation of the mean; namely the observed excess return need not be the true excess return. Even though means are hard to estimate, though, this bias is much smaller than the 
effect on the volatility (by definition, rare events have a large effect on higher-order moments). The majority of the observed premium reflects the fact that announcement days are, in fact, riskier. That is, most of the observed premium reflects the higher population risk premium on announcement days. To summarize, the investor's firstorder condition necessarily imposes a link between risk and return. However, the data does not necessarily capture that risk and return.

\section{Conclusion}

This paper builds a model that explains a strong relation between expected returns and betas on announcement days, but a weak relation on other days. The model simultaneously explains this finding, the high expected return on announcement days, and the fact that measured risk is the same on both types of days.

The mechanism by which the model can explain these facts is one of rare events. We model announcement days as days when news about a latent state is revealed. This news concerns the probability of economic disaster. Most of the time, the news is that the economy is in a good state. The possibility that the news reveals a bad state is what produces the announcement premium. We prove that the model has the qualitative properties found in the data, and we also show, via simulations, that it can quantitatively explain the data.

While our focus in this paper is on macro-announcements, our the methodology can be applied to scheduled announcements more generally, and understanding the rich array of empirical facts that the announcement literature has uncovered. 


\section{A Solving the representative agent's value function}

For a process $X_{t}$, define $\overline{\mathcal{J}}\left(X_{t}\right)$ as follows:

$$
\overline{\mathcal{J}}\left(X_{t}\right)=E_{\nu}\left[X_{t}-X_{t^{-}} \mid d N_{t}=1\right]
$$

Furthermore, define the vector Brownian motion $d B_{t}=\left[d B_{C t}, d B_{\lambda t}\right]^{\top}$.

\section{A.1 Continuation Value}

Lemma A.1. In equilibrium, the representative agent's continuation value equals

$$
J\left(C_{t}, p_{t}, \lambda_{2 t}, \tau ; p_{0 t}\right)=\frac{1}{1-\gamma} C_{t}^{1-\gamma} I\left(p_{t}, \lambda_{2 t}, \tau ; p_{0 t}\right)^{1-\gamma}
$$

with

$$
I\left(p_{t}, \lambda_{2 t}, \tau ; p_{0 t}\right)=\exp \left\{a\left(\tau ; p_{0 t}\right)+b_{p} p_{t}+b_{\lambda} \lambda_{2 t}\right\}
$$

and

$$
\begin{aligned}
& b_{p}=\frac{\left(\lambda^{B}-\lambda^{G}\right) E_{\nu}\left[e^{(1-\gamma) Z_{t}}-1\right]}{(1-\gamma)\left(\beta+\eta_{G B}+\eta_{B G}\right)}, \\
& b_{\lambda}=\frac{1}{(1-\gamma) \sigma_{\lambda}^{2}}\left(\beta+\kappa-\sqrt{(\beta+\kappa)^{2}-2 \sigma_{\lambda}^{2}\left[E_{\nu} e^{(1-\gamma) Z_{t}}-1\right]}\right),
\end{aligned}
$$

for $p_{t} \in[0,1], \lambda_{2 t} \in[0, \infty), \tau \in[0, T), p_{0 t} \in\{0,1\}$ and where a defined as

$$
\begin{aligned}
a\left(\tau ; p_{0 t}\right)=\zeta_{p_{0 t}} e^{\beta \tau}+ & \\
& \frac{1}{\beta}\left(\mu_{C}-\frac{1}{2} \gamma \sigma^{2}+b_{p} \eta_{G B}+b_{\lambda} \kappa \bar{\lambda}_{2}+\frac{\lambda^{G}}{1-\gamma}\left[E_{\nu} e^{(1-\gamma) Z_{t}}-1\right]\right) .
\end{aligned}
$$

The constant terms $\zeta_{0}$ and $\zeta_{1}$ solve

$$
\begin{aligned}
& e^{(1-\gamma)\left(\zeta_{0} e^{\beta T}+b_{p} p^{G}\right)}=p^{G} e^{(1-\gamma)\left(\zeta_{1}+b_{p}\right)}+\left(1-p^{G}\right) e^{(1-\gamma) \zeta_{0}} \\
& e^{(1-\gamma)\left(\zeta_{1} e^{\beta T}+b_{p} p^{B}\right)}=p^{B} e^{(1-\gamma)\left(\zeta_{1}+b_{p}\right)}+\left(1-p^{B}\right) e^{(1-\gamma) \zeta_{0}},
\end{aligned}
$$

with $p^{G}$ and $p^{B}$ defined by 13 .

Proof. Conjecture that the continuation value $V_{t}$ is a function $J\left(C_{t}, p_{t}, \lambda_{2 t}, \tau ; p_{0 t}\right)$, 
where $\tau=t \bmod T$. Note that $p_{0 t}$ is a discrete variable that changes only upon announcements. For $t \in \mathcal{N}$, the Hamilton-Jacobi-Bellman equation applies and

$$
\begin{aligned}
& f\left(C_{t}, J_{t}\right)+\frac{\partial J}{\partial \tau}+\frac{\partial J}{\partial C} C_{t} \mu_{C}+ \frac{\partial J}{\partial p}\left[-p_{t}\left(\eta_{G B}+\eta_{B G}\right)+\eta_{G B}\right]-\frac{\partial J}{\partial \lambda} \kappa\left(\lambda_{2 t}-\bar{\lambda}_{2}\right) \\
&+\frac{1}{2} \frac{\partial^{2} J}{\partial C^{2}} C_{t}^{2} \sigma^{2}+\frac{1}{2} \frac{\partial^{2} J}{\partial \lambda^{2}} \lambda_{2 t} \sigma_{\lambda}^{2} \\
&+\left(p_{t} \lambda^{B}+\left(1-p_{t}\right) \lambda^{G}+\lambda_{2 t}\right) \overline{\mathcal{J}}\left(J\left(C_{t}, p_{t}, \lambda_{2 t}, \tau ; p_{0 t^{-}}\right)\right)=0 .
\end{aligned}
$$

Conjecture a solution to A.6 of the form

$$
J\left(C_{t}, p_{t}, \lambda_{2 t}, \tau ; p_{0 t^{-}}\right)=\frac{1}{1-\gamma} C_{t}^{1-\gamma} I\left(p_{t},, \lambda_{2 t}, \tau ; p_{0 t}\right)^{1-\gamma},
$$

where

$$
I\left(p_{t}, \lambda_{2 t}, \tau ; p_{0 t}\right)=e^{a\left(\tau ; p_{0 t}\right)+b_{p} p+b_{\lambda} \lambda_{2 t}} .
$$

Equation A.8 implies

$$
\frac{\overline{\mathcal{J}}\left(J\left(C_{t}, p_{t}, \lambda_{2 t}, \tau ; p_{0 t}\right)\right)}{J\left(C_{t^{-}}, p_{t^{-}}, \lambda_{2 t^{-}}, \tau^{-} ; p_{0 t^{-}}\right)}=E_{\nu}\left[e^{(1-\gamma) Z_{t}}-1\right]
$$

Substituting A.7 into A.6 and dividing both sides by $J$, we obtain

$$
\begin{aligned}
& -\beta(1-\gamma)\left[a\left(\tau ; p_{0 t}\right)+b_{p} p_{t}+b_{\lambda} \lambda_{2 t}\right] \\
& +(1-\gamma) \frac{d a}{d \tau}\left(\tau ; p_{0 t}\right)+(1-\gamma) \mu_{C}+(1-\gamma) b_{p}\left[-p_{t}\left(\eta_{G B}+\eta_{B G}\right)+\eta_{G B}\right]-(1-\gamma) b_{\lambda} \kappa\left(\lambda_{2 t}-\bar{\lambda}_{2}\right) \\
& \quad-\frac{1}{2} \gamma(1-\gamma) \sigma^{2}+\frac{1}{2}(1-\gamma)^{2} b_{\lambda}^{2} \sigma_{\lambda}^{2} \lambda_{2 t} \\
& \quad+p_{t}\left(\lambda^{B}-\lambda^{G}\right) E_{\nu}\left[e^{(1-\gamma) Z_{t}}-1\right]+\lambda^{G} E_{\nu}\left[e^{(1-\gamma) Z_{t}}-1\right]+\lambda_{2 t}\left[E_{\nu} e^{(1-\gamma) Z_{t}}-1\right]=0 .
\end{aligned}
$$

Collecting coefficients on $p_{t}$ and on $\lambda_{2 t}$, we obtain

$$
\begin{array}{r}
-\beta(1-\gamma) b_{p}-(1-\gamma) b_{p}\left(\eta_{G B}+\eta_{B G}\right)+\left(\lambda^{B}-\lambda^{G}\right) E_{\nu}\left[e^{(1-\gamma) Z_{t}}-1\right]=0 \\
-\beta(1-\gamma) b_{\lambda}-(1-\gamma) b_{\lambda} \kappa+\frac{1}{2}(1-\gamma)^{2} b_{\lambda}^{2} \sigma_{\lambda}^{2}+E_{\nu}\left[e^{(1-\gamma) Z_{t}}-1\right]=0 .
\end{array}
$$

The equation for $b_{p}$ in the text follows. 
We also have the following quadratic function of $b_{\lambda}$ :

$$
\frac{1}{2}(1-\gamma) \sigma_{\lambda}^{2} b_{\lambda}^{2}-(\beta+\kappa) b_{\lambda}+\frac{1}{1-\gamma} E_{\nu}\left[e^{(1-\gamma) Z_{t}}-1\right]=0
$$

which has solution 15

$$
b_{\lambda}=\frac{1}{(1-\gamma) \sigma_{\lambda}^{2}}\left(\beta+\kappa-\sqrt{(\beta+\kappa)^{2}-2 \sigma_{\lambda}^{2} E_{\nu}\left[e^{(1-\gamma) Z_{t}}-1\right]}\right) \text {. }
$$

Finally we solve $a\left(\tau ; p_{0 t}\right)$. Collecting constant terms gives the ODE

$$
\begin{aligned}
& -\beta(1-\gamma) a\left(\tau ; p_{0 t}\right)+(1-\gamma) \frac{d a}{d \tau}\left(\tau ; p_{0 t}\right) \\
+ & (1-\gamma) \mu_{C}+(1-\gamma) b_{p} \eta_{G B}+(1-\gamma) b_{\lambda} \kappa \bar{\lambda}_{2}-\frac{1}{2} \gamma(1-\gamma) \sigma^{2}+\lambda^{G} E_{\nu}\left[e^{(1-\gamma) Z_{t}}-1\right]=0,
\end{aligned}
$$

which is equivalent to

$$
\frac{d a}{d \tau}\left(\tau ; p_{0 t}\right)=\beta a\left(\tau ; p_{0 t}\right)-\mu_{C}+\frac{1}{2} \gamma \sigma^{2}-b_{p} \eta_{G B}-b_{\lambda} \kappa \bar{\lambda}_{2}-\frac{\lambda^{G}}{1-\gamma} E_{\nu}\left[e^{(1-\gamma) Z_{t}}-1\right]
$$

Equation A.14 implies a general form for $a\left(\tau ; p_{0 t}\right)$ :

$$
\begin{aligned}
& a\left(\tau ; p_{0 t}\right)= \\
& \quad \zeta_{p_{0 t}} e^{\beta \tau}+\frac{1}{\beta}\left(\mu_{C}-\frac{1}{2} \gamma \sigma^{2}+b_{p} \eta_{G B}+b_{\lambda} \kappa \bar{\lambda}+\frac{\lambda^{G}}{1-\gamma} E_{\nu}\left[e^{(1-\gamma) Z_{t}}-1\right]\right),
\end{aligned}
$$

where $\zeta_{p_{0 t}} \in\left\{\zeta_{0}, \zeta_{1}\right\}$ for as yet undetermined coefficients $\zeta_{0}$ and $\zeta_{1}$.

To obtain $\zeta_{0}$ and $\zeta_{1}$, we require boundary conditions for A.14. We obtain these from the optimality condition at announcements. Along the optimal path, continuation value must satisfy

$$
\begin{aligned}
V_{t^{-}} & =E_{t^{-}}\left[\int_{t}^{\infty} f\left(C_{s}, V_{s}\right) d s\right] \\
& =E_{t^{-}}\left[V_{t}\right] .
\end{aligned}
$$

Equation A.16 is trivial except on announcements. Upon announcements $(t \in \mathcal{A})$, A.16 gives us the required boundary conditions. First note that, by definition of $\mathcal{A}$

\footnotetext{
${ }^{15}$ See $\overparen{\text { Tsai and Wachter }}(2015)$ for details about choosing the solution to $b_{\lambda}$.
} 
and of $\tau$,

$$
\lim _{\tau \rightarrow T} J\left(C_{t^{-}}, p_{t^{-}}, \lambda_{2, t^{-}}, \tau ; p_{0 t^{-}}\right)=E_{t^{-}}\left[J\left(C_{t}, p_{t}, \lambda_{2 t}, 0 ; p_{0 t}\right)\right]
$$

Furthermore, because $C_{t}$ and $\lambda_{2 t}$ are continuous with probability 1 ,

$$
\lim _{\tau \rightarrow T} J\left(C_{t}, p_{t^{-}}, \lambda_{2 t}, \tau ; p_{0 t^{-}}\right)=E_{t^{-}}\left[J\left(C_{t}, p_{t}, \lambda_{2 t}, 0 ; p_{0 t}\right)\right]
$$

Equation A.17, together with the form of $I$ and Lemma 1, restricts $a\left(\tau ; p_{0 t}\right)$. Namely,

$$
\lim _{\tau \rightarrow T} \exp \left\{(1-\gamma)\left(a\left(\tau ; p_{0 t^{-}}\right)+b_{p} p_{t^{-}}\right)\right\}=E_{t^{-}}\left[\exp \left\{(1-\gamma)\left(a\left(0 ; p_{0 t}\right)+b_{p} p_{t}\right\}\right]\right.
$$

for $t \in \mathcal{A}$. Cancelling out the constant term in $a(\cdot, \cdot)$ implies

$$
\exp \left\{(1-\gamma)\left(\zeta_{p_{0 t^{-}}} e^{\beta T}+b_{p} p_{t^{-}}\right)\right\}=E_{t^{-}}\left[\exp \left\{(1-\gamma) \zeta_{p_{0 t}}+b_{p} p_{t}\right\}\right], \quad t \in \mathcal{A}
$$

Immediately following the announcement $p_{t}=p_{0 t} \in\{0,1\}$. Therefore,

$$
\exp \left\{(1-\gamma)\left(\zeta_{p_{0 t^{-}}} e^{\beta T}+b_{p} p_{t^{-}}\right)\right\}=\left(1-p_{t^{-}}\right) e^{(1-\gamma) \zeta_{0}}+p_{t^{-}} e^{(1-\gamma) \zeta_{1}+b_{p}}
$$

Applying (A.18) at $p_{0 t^{-}}=0$ and $p_{0 t^{-}}=1$ implies A.5), uniquely determining $\zeta_{0}$ and $\zeta_{1}$.

\section{A.2 The state price density}

Lemma A.2. The process $\pi_{t}$ is characterized by

$$
\pi_{t}=\beta \exp \left\{\int_{0}^{t} \frac{\partial}{\partial V} f\left(C_{s}, V_{s}\right) d s\right\} C_{t}^{-\gamma} I\left(p_{t}, \lambda_{2 t}, \tau ; p_{0 t}\right)^{1-\gamma}
$$

where $I\left(p_{t}, p_{0 t}, \lambda_{2 t}, \tau\right)$ is defined by (A.3).

Proof. Duffie and Skiadas (1994) show that

$$
\pi_{t}=\exp \left\{\int_{0}^{t} \frac{\partial}{\partial V} f\left(C_{s}, V_{s}\right) d s\right\} \frac{\partial}{\partial C} f\left(C_{t}, V_{t}\right) .
$$


The form of $f$ implies

$$
\begin{aligned}
\frac{\partial}{\partial C} f\left(C_{t}, V_{t}\right) & =\beta(1-\gamma) \frac{V_{t}}{C_{t}} \\
& =\beta(1-\gamma) \frac{(1-\gamma)^{-1}\left(C_{t}\right)^{1-\gamma} I\left(p_{t}, \lambda_{2 t}, \tau ; p_{0 t}\right)^{1-\gamma}}{C_{t}} \\
& =\beta C_{t}^{-\gamma} I\left(p_{t}, \lambda_{2 t}, \tau ; p_{0 t}\right)^{1-\gamma} .
\end{aligned}
$$

Combining (A.20) and (A.21) implies

$$
\pi_{t}=\beta \exp \left\{\int_{0}^{t} \frac{\partial}{\partial V} f\left(C_{s}, V_{s}\right) d s\right\} C_{t}^{-\gamma} I\left(p_{t}, \lambda_{2 t}, \tau ; p_{0 t}\right)^{1-\gamma}
$$

Proof of Theorem 1. For this proof, assume $t \in \mathcal{N}$. Ito's Lemma and Lemma A.2 imply

$$
\frac{d \pi_{t}}{\pi_{t^{-}}}=\mu_{\pi t} d t+\sigma_{\pi t} d B_{t}+\frac{\pi_{t}-\pi_{t^{-}}}{\pi_{t^{-}}} d N_{t}
$$

for a scalar process $\mu_{\pi t}$ and a $1 \times 2$ vector process $\sigma_{\pi t}{ }^{16}$ It follows from A.20 and Ito's Lemma that

$$
\sigma_{\pi t}=\left[-\gamma \sigma,(1-\gamma) b_{\lambda} \sigma_{\lambda} \sqrt{\lambda_{2 t}}\right]
$$

and that

$$
\frac{\pi_{t}-\pi_{t^{-}}}{\pi_{t^{-}}}=e^{-\gamma Z_{t}}-1
$$

It follows from no-arbitrage that

$$
E_{t^{-}}\left[\frac{d \pi_{t}}{\pi_{t^{-}}}\right]=-r_{t^{-}} d t
$$

It then follows from the definition of an intensity that

$$
E_{t^{-}}\left[d \pi_{t}\right]=\left(\mu_{\pi t} \pi_{t^{-}}+\left(\bar{\lambda}_{1}\left(p_{t}\right)+\lambda_{2 t}\right) \overline{\mathcal{J}}\left(\pi_{t}\right)\right) d t
$$

implying

$$
\mu_{\pi t}=-r_{t}-\left(\bar{\lambda}_{1}\left(p_{t}\right)+\lambda_{2 t}\right) \frac{\overline{\mathcal{J}}\left(\pi_{t}\right)}{\pi_{t^{-}}},
$$

\footnotetext{
${ }^{16}$ Lemma A.2 also implies the continuity of $\mu_{\pi t}$ and $\sigma_{\pi t}$ on non-announcement dates. This allows us to use $t$ rather than $t^{-}$to subscript these variables in $\mathrm{A} .22$ and elsewhere.
} 
where $r_{t}=r_{t^{-}}$because $\mu_{\pi t}, \lambda_{2 t}$ are continuous and $\frac{\overline{\mathcal{J}}\left(\pi_{t}\right)}{\pi_{t^{-}}}$is constant.

Proof of Theorem 2. Note that

$$
\begin{aligned}
\frac{\partial}{\partial V} f\left(C_{t}, V_{t}\right) & =\frac{\partial}{\partial V}\left(\beta(1-\gamma) V_{t} \log C_{t}-\beta V_{t} \log \left[(1-\gamma) V_{t}\right]\right) \\
& =\beta(1-\gamma) \log C_{t}-\beta \log \left[(1-\gamma) V_{t}\right]-\beta \\
& =-\beta\left\{1+(1-\gamma)\left[a\left(\tau ; p_{0 t}\right)+b_{p} p+b_{\lambda} \lambda_{2 t}\right]\right\}
\end{aligned}
$$

It follows from A.19 and Ito's Lemma that

$$
\begin{aligned}
\mu_{\pi t}= & \left\{-\beta\left[1+(1-\gamma) a\left(\tau ; p_{0 t}\right)+(1-\gamma) b_{p} p_{t}+(1-\gamma) b_{\lambda} \lambda_{2 t}\right]+(1-\gamma) \frac{\partial a}{\partial \tau}\right\} d t \\
& -\gamma \mu_{C} d t+(1-\gamma) b_{p}\left[-p_{t} \eta_{B G}+(1-p) \eta_{G B}\right] d t-(1-\gamma) b_{\lambda} \kappa\left(\lambda_{2 t}-\bar{\lambda}_{2}\right) d t \\
& +\frac{1}{2} \gamma(\gamma+1) \sigma^{2} d t+\frac{1}{2}(1-\gamma)^{2} b_{\lambda}^{2} \sigma_{\lambda}^{2} \lambda_{2 t} d t
\end{aligned}
$$

Collecting terms and applying the equations for $a\left(\tau ; p_{0 t}\right), b_{p}$ and $b_{\lambda}$ yields

$$
\mu_{\pi t}=-\left(\beta+\mu_{C}-\gamma \sigma^{2}+\left(\bar{\lambda}_{1}\left(p_{t}\right)+\lambda_{2 t}\right)\left[E_{\nu} e^{(1-\gamma) Z_{t}}-1\right]\right) d t
$$

The result then follows from A.25.

Proof of Theorem 3 . Consider $t \in \mathcal{A}$, namely announcement times. With probability 1 , a disaster does not coincide with an announcement. Therefore, it follows from A.19 that

$$
\frac{\pi_{t}}{\pi_{t^{-}}}=\lim _{\tau \rightarrow T} \frac{I\left(p_{t}, \lambda_{2 t}, 0 ; p_{0 t}\right)}{I\left(p_{t^{-}}, \lambda_{2 t}, \tau ; p_{0 t^{-}}\right)}=\lim _{\tau \rightarrow T} \frac{e^{(1-\gamma)\left(a\left(0 ; p_{0 t}\right)+b_{p} p_{t}\right)}}{e^{(1-\gamma)\left(a\left(\tau ; p_{0 t^{-}}\right)+b_{p} p_{t^{-}}\right)}}
$$

The first equality holds on a set of outcomes of measure zero ${ }^{17}$ We use the fact that $\lambda_{2 t}$ is continuous. The second inequality follows from A.8. The result then follows directly from the definition of $a(\cdot ; \cdot)$ in A.4.

Proof of Lemma 2. We want to show $\zeta_{0}>\zeta_{1}+b_{p}$. We prove this by contradiction.

Suppose that

$$
\zeta_{0} \leq \zeta_{1}+b_{p}
$$

\footnotetext{
${ }^{17}$ There is zero probability that a disaster occurs on an announcement date.
} 
Recall the following pair of equations which determine $\zeta_{0}$ and $\zeta_{1}$ :

$$
\begin{aligned}
& e^{(1-\gamma)\left(\zeta_{0} e^{\beta T}+b_{p} p^{G}\right)}=p^{G} e^{(1-\gamma)\left(\zeta_{1}+b_{p}\right)}+\left(1-p^{G}\right) e^{(1-\gamma) \zeta_{0}} \\
& e^{(1-\gamma)\left(\zeta_{1} e^{\beta T}+b_{p} p^{B}\right)}=p^{B} e^{(1-\gamma)\left(\zeta_{1}+b_{p}\right)}+\left(1-p^{B}\right) e^{(1-\gamma) \zeta_{0}}
\end{aligned}
$$

The expressions on the left hand side of A.28) are weighted averages of $e^{(1-\gamma)\left(\zeta_{1}+b_{p}\right)}$ and $e^{(1-\gamma) \zeta_{0}}$ with weights between 0 and 1 . Thus they must lie between these two terms.

First assume that $\gamma<1$. Because the exponential is an increasing function, assumption A.27) implies

$$
\begin{aligned}
\zeta_{0} & \leq \zeta_{0} e^{\beta T}+b_{p} p^{G} \\
\zeta_{1} e^{\beta T}+b_{p} p^{B} & \leq \zeta_{1}+b_{p}
\end{aligned}
$$

Furthermore, if $\gamma>1$, then (A.27) and (A.28) imply

$$
\begin{aligned}
(1-\gamma) \zeta_{0} & \geq(1-\gamma)\left(\zeta_{0} e^{\beta T}+b_{p} p^{G}\right) \\
(1-\gamma)\left(\zeta_{1} e^{\beta T}+b_{p} p^{B}\right) & \geq(1-\gamma)\left(\zeta_{1}+b_{p}\right),
\end{aligned}
$$

which also implies A.29

It follows that

$$
\begin{aligned}
& \zeta_{0}\left(1-e^{\beta T}\right) \leq b_{p} p^{G}<0 \\
& \zeta_{1}\left(e^{\beta T}-1\right) \leq b_{p}\left(1-p^{B}\right)<0,
\end{aligned}
$$

because $b_{p}<0$. Therefore $\zeta_{0}>0$ and $\zeta_{1}<0$, contradicting (A.27).

The following result is of interest in its own right, and will be useful in proving results for equity in what follows.

Lemma A.3. Let $\tilde{p}^{B}$ be the risk-neutral probability of a negative announcement, just prior to the announcement occurring, provided that the previous announcement was negative, and $\tilde{p}^{G}$ be the analogous quantity, provided that the previous announcement 
was positive. Then

$$
\tilde{p}^{B}>\tilde{p}^{G} .
$$

Proof. It follows from (10), applied in the limit as $\tau \rightarrow T$, that $p^{B}>p^{G}$. This is intuitive: because states are persistent, if the previous announcement revealed a negative state, it is more likely that the next announcement will also reveal a negative state than if the previous announcement were positive.

Define the notation

$$
\begin{aligned}
\pi^{G} & =e^{(1-\gamma) \zeta_{0}} \\
\pi^{B} & =e^{(1-\gamma)\left(\zeta_{1}+b_{p}\right)} \\
\pi^{0, G} & =e^{(1-\gamma)\left(\zeta_{0} e^{\beta T}+b_{p} p^{G}\right)} \\
\pi^{0, B} & =e^{(1-\gamma)\left(\zeta_{1} e^{\beta T}+b_{p} p^{B}\right)} .
\end{aligned}
$$

It follows from (16) that

$$
\begin{aligned}
& \tilde{p}^{G}=p^{G} \frac{\pi^{B}}{\pi^{0, G}} \\
& \tilde{p}^{B}=p^{B} \frac{\pi^{B}}{\pi^{0, B}} .
\end{aligned}
$$

First consider the case of $\gamma>1$. We want to show that

$$
\frac{p_{B}}{p_{G}}>\frac{\pi^{0, B}}{\pi^{0, G}}=\frac{p^{B}+\frac{\pi^{G}}{\pi^{B}-\pi^{G}}}{p^{G}+\frac{\pi^{G}}{\pi^{B}-\pi^{G}}}
$$

The second inequality follows from (18), or equivalently, $\pi_{t^{-}}=E_{t^{-}} \pi_{t}$. Moreover, Lemma 2 implies $\pi^{B}>\pi^{G}$. Because $\frac{p^{B}+x}{p^{G}+x}$ is a decreasing function of $x$, the result follows.

Now consider $\gamma<1$. Because $\pi^{G}>\pi^{B}, \pi^{0, G}>\pi^{0, B}$. Thus

$$
\frac{\pi^{0, B}}{\pi^{0, G}}<1<\frac{p^{B}}{p^{G}} .
$$




\section{B Pricing equity}

Appendix B.1 derives result for equity strips (namely a claim to a dividend paid at a single point in time). Appendix B.2 uses these results to derive results for dividend streams. We suppress the $j$ subscript when not essential for clarity.

\section{B.1 Pricing equity strips}

We first derive the no-arbitrage condition on intervals without announcements.

Lemma B.1. Let $H_{t}$ denote the time-t price of a dividend $D_{t^{*}}$ with $t^{*} \geq t$, such that the distribution of $D_{t^{*}} / D_{t}$ is determined by the state vector $p_{t}, p_{0 t}, \lambda_{2 t}$. Define $s=t^{*}-t$ and $\tau=t \bmod T$. Then

$$
H_{t}=H\left(D_{t}, p_{t}, \lambda_{2 t}, \tau, s ; p_{0 t}\right)=E_{t}\left[\frac{\pi_{t^{*}}}{\pi_{t}} D_{t^{*}}\right],
$$

Moreover, for $t \in \mathcal{N}, H_{t}$ satisfies

$$
\frac{d H_{t}}{H_{t^{-}}}=\mu_{H t} d t+\sigma_{H t} d B_{t}+\frac{H_{t}-H_{t^{-}}}{H_{t^{-}}} d N_{t},
$$

with $\mu_{H t}=\mu_{H}\left(p_{t}, \lambda_{2 t}, \tau, s ; p_{0 t}\right)$ and $\sigma_{H t}=\sigma_{H}\left(p_{t}, \lambda_{2 t}, \tau, s ; p_{0 t}\right)$, satisfying

$$
\mu_{H t}+\mu_{\pi t}+\sigma_{H t} \sigma_{\pi t}^{\top}+\left(\bar{\lambda}_{1}\left(p_{t}\right)+\lambda_{2 t}\right) \frac{\overline{\mathcal{J}}\left(H_{t} \pi_{t}\right)}{H_{t^{-}} \pi_{t^{-}}}=0 .
$$

Proof. Equation B.1 follows from the absence of arbitrage and the Markov property for the dividend process $D_{t}$ and the state-price density $\pi_{t}$. Given that $H_{t} / D_{t}$ is a function of the state variables, (B.2) follows from Ito's Lemma. Moreover, B.1) directly implies that $\pi_{t} H_{t}$ is a martingale.

Define

$$
u_{l}=\inf \left\{t: N_{t}=l\right\},
$$

as the arrival time of the $l^{\text {th }}$ Poisson arrival. Consider $t \in \mathcal{N}$ and chose $\Delta t$ sufficiently small so that the interval $[t, t+\Delta t]$ does not contain an announcement. It follows from 
(B.2) that

$$
\begin{array}{r}
H_{t+\Delta t} \pi_{t+\Delta t}=H_{t} \pi_{t}+\int_{t}^{t+\Delta t} \pi_{u} H_{u}\left(\mu_{H u}+\mu_{\pi u}+\sigma_{H u} \sigma_{\pi u}^{\top}\right) d u+\int_{t}^{t+\Delta t} \pi_{u} H_{u}\left(\sigma_{H u}+\sigma_{\pi u}\right) d B_{u} \\
+\sum_{t<u_{l} \leq t+\Delta t}\left(\pi_{u_{l}} H_{u_{l}}-\pi_{u_{l^{-}}} H_{u_{l^{-}}}\right) . \quad \text { (B.5) }
\end{array}
$$

Rewriting, we have:

$$
\begin{gathered}
H_{t+\Delta t} \pi_{t+\Delta t}=H_{t} \pi_{t}+\underbrace{\int_{t}^{t+\Delta t} \pi_{u} H_{u}\left(\mu_{H u}+\mu_{\pi u}+\sigma_{H u} \sigma_{\pi u}^{\top}+\left(\bar{\lambda}_{1}\left(p_{u}\right)+\lambda_{2 u}\right) \frac{\overline{\mathcal{J}}\left(H_{u} \pi_{u}\right)}{H_{u^{-}} \pi_{u^{-}}}\right) d u}_{(1)} \\
+\underbrace{\int_{t}^{t+\Delta t} \pi_{u} H_{u}\left(\sigma_{H u}+\sigma_{\pi u}\right) d B_{u}}_{(2)} \\
+\underbrace{\text { B. } 6)}_{\sum_{t<u_{l} \leq t+\Delta t}\left(\pi_{u_{l}} H_{u_{l}}-\pi_{u_{l^{-}}} H_{u_{l^{-}}}\right)-\int_{t}^{t+\Delta t}\left(\bar{\lambda}_{1}\left(p_{u}\right)+\lambda_{2 u}\right) \overline{\mathcal{J}}\left(H_{u} \pi_{u}\right) d u}
\end{gathered}
$$

Since $H_{t} \pi_{t}$ is a martingale, the time- $t$ expectation of $H_{t+\Delta t} \pi_{t+\Delta t}$ must be $H_{t} \pi_{t}$. In (B.6), (2) and (3) have zero expectation, so that the integrand in (1) must be zero. We obtain (B.3).

Proof of Theorem 4. Define $H_{t}$ as in Lemma B.1. Conjecture that $H_{t}$ takes the form (23) for as-yet unspecified functions $a_{\phi}\left(\tau, s ; p_{0}\right), b_{\phi p}(s)$ and $b_{\phi \lambda}(s)$. No-arbitrage implies the following boundary condition for the zero-maturity claim:

$$
H\left(D, p, \lambda_{2}, \tau, 0 ; p_{0}\right)=D
$$

Thus

$$
a_{\phi}\left(\tau, 0 ; p_{0}\right)=b_{\phi p}(0)=b_{\phi \lambda}(0)=0
$$

Consider $t \in \mathcal{N}$. Define $\mu_{H t}$ and $\sigma_{H t}$ as in Lemma B.1. Applying Ito's Lemma to 
the conjecture 23 implies

$$
\begin{gathered}
\mu_{H t}=\mu_{D}+\frac{\partial a_{\phi}}{\partial \tau}-\frac{\partial a_{\phi}}{\partial s}-\frac{\partial b_{\phi p}}{\partial s} p_{t}-\frac{\partial b_{\phi \lambda}}{\partial s} \lambda_{2 t}+\frac{1}{2} b_{\phi \lambda}(s)^{2} \sigma_{\lambda}^{2} \lambda_{2 t} \\
+b_{\phi p}\left[-p_{t} \eta_{B G}+(1-p) \eta_{G B}\right]+b_{\phi \lambda}(s)\left[-\kappa\left(\lambda_{2 t}-\bar{\lambda}_{2}\right)\right] \\
=\mu_{D}+\frac{\partial a_{\phi}}{\partial \tau}-\frac{\partial a_{\phi}}{\partial s}+b_{\phi p} \eta_{G B}+b_{\phi \lambda}(s) \kappa \bar{\lambda}_{2} \\
+\left(-\frac{\partial b_{\phi p}}{\partial s}-b_{\phi p}\left(\eta_{B G}+\eta_{G B}\right)\right) p_{t}+\left(-\frac{\partial b_{\phi \lambda}}{\partial s}+\frac{1}{2} b_{\phi \lambda}(s)^{2} \sigma_{\lambda}^{2}+\kappa b_{\phi \lambda}(s)\right) \lambda_{2 t}
\end{gathered}
$$

and

$$
\sigma_{H t}=\left[\sigma, b_{\phi \lambda}(s) \sigma_{\lambda} \sqrt{\lambda_{2 t}}\right]
$$

Moreover, $\pi_{t}$ satisfies A.22 with

$$
\mu_{\pi t}=-\left(\beta+\mu-\gamma \sigma^{2}\right)-\left(\bar{\lambda}_{1}\left(p_{t}\right)+\lambda_{2 t}\right) E_{\nu}\left[e^{(1-\gamma) Z_{t}}-1\right]
$$

and $\sigma_{\pi t}$ given in A.23, as Appendix A shows. Finally, 23 and A.24 imply

$$
\frac{\overline{\mathcal{J}}\left(H_{t} \pi_{t}\right)}{H_{t^{-}} \pi_{t^{-}}}=E_{\nu}\left[e^{(\phi-\gamma) Z_{t}}-1\right] \text {. }
$$

It follows that

$$
\begin{aligned}
\mu_{H t}+ & \mu_{\pi t}+\sigma_{H t}^{\top} \sigma_{\pi t}+\left(\bar{\lambda}_{1}\left(p_{t}\right)+\lambda_{2 t}\right) \frac{\overline{\mathcal{J}}\left(H_{t} \pi_{t}\right)}{H_{t^{-}} \pi_{t^{-}}} \\
=\mu_{D}+ & \frac{\partial a_{\phi}}{\partial \tau}-\frac{\partial a_{\phi}}{\partial s}+b_{\phi p}(s) \eta_{G B}+b_{\phi \lambda}(s) \kappa \bar{\lambda}_{2} \\
+ & \left(-\frac{\partial b_{\phi p}}{\partial s}-b_{\phi p}(s)\left(\eta_{B G}+\eta_{G B}\right)\right) p_{t}+\left(-\frac{\partial b_{\phi \lambda}}{\partial s}+\frac{1}{2} b_{\phi \lambda}(s)^{2} \sigma_{\lambda}^{2}-\kappa b_{\phi \lambda}(s)\right) \lambda_{2 t} \\
& -\left(\beta+\mu_{C}-\gamma \sigma^{2}\right)-\left[\lambda^{G}+p_{t}\left(\lambda^{B}-\lambda^{G}\right)\right] E_{\nu}\left[e^{(1-\gamma) Z_{t}}-1\right]-\lambda_{2 t} E_{\nu}\left[e^{(1-\gamma) Z_{t}}-1\right] \\
& -\gamma \sigma^{2}+(1-\gamma) b_{\lambda} b_{\phi \lambda}(s) \sigma_{\lambda}^{2} \lambda_{2 t} \\
=\frac{\partial a_{\phi}}{\partial \tau}- & \frac{\partial a_{\phi}}{\partial s}-\beta-\mu_{C}+\mu_{D}+\lambda^{G} E_{\nu}\left[e^{(\phi-\gamma) Z_{t}}-e^{(1-\gamma) Z_{t}}\right]+\kappa \bar{\lambda}_{2} b_{\phi \lambda}(s) \\
+ & \left\{-\frac{\partial b_{\phi p}}{\partial s}-\left(\eta_{B G}+\eta_{G B}\right) b_{\phi p}(s)+\left(\lambda^{B}-\lambda^{G}\right) E_{\nu}\left[e^{(\phi-\gamma) Z_{t}}-e^{(1-\gamma) Z_{t}}\right]\right\} p_{t} \\
& +\left\{-\frac{\partial b_{\phi \lambda}}{\partial s}+\frac{1}{2} b_{\phi \lambda}(s)^{2} \sigma_{\lambda}^{2}-\kappa b_{\phi \lambda}(s)+(1-\gamma) b_{\lambda} b_{\phi \lambda}(s) \sigma_{\lambda}^{2}+E_{\nu}\left[e^{(\phi-\gamma) Z_{t}}-e^{(1-\gamma) Z_{t}}\right]\right\} \lambda_{2 t} .
\end{aligned}
$$


Equation B.3 implies that the left-hand side of $(\mathrm{B} .12)$ is equal to zero. We therefore have the following ordinary differential equations:

$$
\begin{aligned}
-\frac{\partial b_{\phi p}(s)}{\partial s}-\left(\eta_{B G}+\eta_{G B}\right) b_{\phi p}(s)+\left(\lambda^{B}-\lambda^{G}\right) E_{\nu}\left[e^{(\phi-\gamma) Z_{t}}-e^{(1-\gamma) Z_{t}}\right] & =0 \\
-\frac{d b_{\phi \lambda}(s)}{d s}+\frac{1}{2} \sigma_{\lambda}^{2} b_{\phi \lambda}(s)^{2}+\left[(1-\gamma) b_{\lambda} \sigma_{\lambda}^{2}-\kappa\right] b_{\phi \lambda}(s)+E_{\nu}\left[e^{(\phi-\gamma) Z_{t}}-e^{(1-\gamma) Z_{t}}\right] & =0
\end{aligned}
$$

and the partial differential equation

$$
\frac{\partial a_{\phi}}{\partial \tau}-\frac{\partial a_{\phi}}{\partial s}=\beta+\mu_{C}-\mu_{D}-\lambda^{G} E_{\nu}\left[e^{(\phi-\gamma) Z_{t}}-e^{(1-\gamma) Z_{t}}\right]-\kappa \bar{\lambda}_{2} b_{\phi \lambda}(s) .
$$

Then (24) uniquely solves (B.13) together with the boundary condition (B.7). Moreover, (B.15) and (B.7) ensure that that $a_{\phi}$ takes the form (26).

Finally, we solve for the function $h$. Recall that $a\left(\tau, 0 ; p_{0}\right)=0$, for all $\tau \in[0, T)$. Then, from (26), $h\left(\tau ; p_{0}\right)=0$ for all $\tau \in[0, T)$. However, $h$ is only defined as a function of $\tau+s$. Therefore $h\left(u ; p_{0}\right)=0$, for $u \in[0, T)$.

For $u \geq T$, (27) iteratively determines $h\left(u ; p_{0}\right)$. We now derive (27). Absence of arbitrage and the (almost sure) continuity of $D_{t}, \lambda_{2 t}$ and $s$ imply, for $t \in \mathcal{A}$,

$$
\lim _{\tau \rightarrow T} H\left(D_{t}, p_{t^{-}}, \lambda_{2 t}, \tau, s ; p_{0 t^{-}}\right)=E_{t^{-}}\left[\frac{\pi_{t}}{\pi_{t^{-}}} H\left(D_{t}, p_{t}, \lambda_{2 t}, 0, s ; p_{0 t}\right)\right]
$$

We use (23) to write (B.16) more explicitly as

$$
\lim _{\tau \rightarrow T} \exp \left\{a_{\phi}\left(\tau, s ; p_{0 t^{-}}\right)+b(s) p_{t^{-}}\right\}=E_{t^{-}}\left[\frac{\pi_{t}}{\pi_{t^{-}}} \exp \left\{a_{\phi}\left(0, s ; p_{0 t}\right)+b(s) p_{t}\right\}\right]
$$

Equation 26 and (B.17) then imply the following restriction on $h$ :

$$
\exp \left\{h\left(T+s ; p_{0 t^{-}}\right)+b(s) p_{t^{-}}\right\}=E_{t^{-}}\left[\frac{\pi_{t}}{\pi_{t^{-}}} \exp \left\{h\left(s ; p_{0 t}\right)+b(s) p_{t}\right\}\right]
$$

Defining $u=T+s$ and substituting in for the announcement $\operatorname{SDF} \pi_{t} / \pi_{t^{-}}$from (16) gives us 27.

It remains to show that (27) uniquely characterizes $h$. The discussion above establishes $h\left(u, p_{0}\right)=0$ is the unique solution for $u<T$. We show uniqueness by induction 
of the number of announcements prior to maturity of the asset. Define

$$
n=\left\lfloor\frac{u}{T}\right\rfloor
$$

Assume by induction that $h$ is unique for $u \in[(n-1) T, n T), n \geq 1$. Then, for each $u \in[n T,(n+1) T),(27)$, applied at $p_{0 t^{-}}=0$ and $p_{0 t^{-}}=1$ is a system of two equations in two unknowns. It therefore uniquely pins down $h\left(u ; p_{0}\right)$.

We now sign the effect of an announcement on an equity strip.

Corollary B.1. Assume that $\phi>1$. Then the price of an equity strip with positive maturity increases when the announcement is positive and decreases when the announcement is negative.

Proof. We seek to determine the sign of $H_{t}-H_{t^{-}}$for $t \in \mathcal{A}$.

Using (23), (26), and the almost-sure continuity of all variables around announcements, with the exception of $p_{t}$ and $p_{0 t}$, it suffices to show that

$$
h(s ; 0)>h(s ; 1)+b_{\phi p}(s)
$$

for $s>0$. The reason is that $(\mathrm{B} .19)$ is equivalent to the result that $H_{t}$ is lower for a negative announcement than for a positive announcement. Because $H_{t^{-}}$is a weighted average of these outcomes, it follows that $H_{t}<H_{t^{-}}$when the announcement is positive and $H_{t}>H_{t^{-}}$when the announcement is negative.

When $s<T$, B.19) follows from $h(s ; 1)=h(s ; 0)=0$ and $b_{\phi p}(s)<0$ when $\phi>1$. We now show $(\mathrm{B} .19)$ for general $s>T$ using induction on the number of announcements prior to maturity. Assume the condition holds for $s \in[(n-1) T, n T)$. Using (27) and the definition of the risk-neutral probabilities from Lemma A.3 we have

$$
\begin{aligned}
& e^{h(s ; 0)+b_{\phi p}(s-T) p^{G}}=\tilde{p}^{G} e^{h(s-T ; 1)+b_{\phi p}(s-T)}+\left(1-\tilde{p}^{G}\right) e^{h(s-T ; 0)} \\
& e^{h(s ; 1)+b_{\phi p}(s-T) p^{B}}=\tilde{p}^{B} e^{h(s-T ; 1)+b_{\phi p}(s-T)}+\left(1-\tilde{p}^{B}\right) e^{h(s-T ; 0)}
\end{aligned}
$$

Lemma A.3 shows that $\tilde{p}^{B}>\tilde{p}^{G}$. Therefore, by the induction step

$$
h(s ; 0)+b_{\phi p}(s-T) p^{G}>h(s ; 1)+b_{\phi p}(s-T) p^{B} .
$$


Finally,

$$
\begin{aligned}
h(s ; 0) & >h(s ; 0)+b_{\phi p}(s-T) p^{G} \\
& >h(s ; 1)+b_{\phi p}(s-T) p^{B} \\
& >h(s ; 1)+b_{\phi p}(s-T) \\
& >h(s ; 1)+b_{\phi p}(s) .
\end{aligned}
$$

The last inequality follows because $b_{\phi p}$ is a strictly decreasing function. Therefore (B.19) holds for all $s>0$, which completes the proof.

\section{B.2 Pricing streams of dividends (equity)}

The following Lemma extends Lemma B.1 to the case of an asset paying a stream of dividends.

Lemma B.2. Let $F_{t}=F\left(D_{t}, p_{t}, \lambda_{2 t}, \tau ; p_{0 t}\right)$ denote the time-t price of a future dividend stream $\left\{D_{s}\right\}_{s \in(t, \infty)}$ with growth rates satisfying the Markov property with respect to the state vector $\left(p_{t}, p_{0 t}, \lambda_{2 t}\right)$. Then

$$
F_{t}=E_{t} \int_{t}^{\infty} \frac{\pi_{u}}{\pi_{t}} D_{u} d u
$$

Moreover, for $t$ such that $t$ mod $T \neq 0$, there exist processes $\mu_{F, t}=\mu_{F}\left(p_{t}, \lambda_{2 t}, \tau ; p_{0 t}\right)$ and $\sigma_{F, t}=\sigma_{F}\left(p_{t}, \lambda_{2 t}, \tau ; p_{0 t}\right)$ such that

$$
\frac{d F_{t}}{F_{t^{-}}}=\mu_{F t} d t+\sigma_{F t} d B_{t}+\frac{F_{t}-F_{t^{-}}}{F_{t^{-}}} d N_{t}
$$

that satisfy the no-arbitrage restriction

$$
\mu_{\pi t}+\mu_{F t}+\frac{D_{t}}{F_{t}}+\sigma_{\pi t} \sigma_{F t}^{\top}+\left(\bar{\lambda}_{1}\left(p_{t}\right)+\lambda_{2 t}\right) \frac{\overline{\mathcal{J}}\left(\pi_{t} F_{t}\right)}{\pi_{t^{-}} F_{t^{-}}}=0 .
$$

Proof. Applying (B.1) and interchanging the position of the integral and the expectation, we have

$$
F_{t}=\int_{0}^{\infty} H\left(D_{t}, p_{t}, \lambda_{2 t}, \tau, s ; p_{0 t}\right) d s,
$$

Equation B.21 then follows by Ito's Lemma and the homogeneity of $H$ in $D$. Let $\mu_{H(s), t}=\mu_{H}\left(D_{t}, p_{t}, p_{0 t}, \lambda_{2 t}, \tau, s\right)$ and $\sigma_{H(s), t}=\sigma_{H}\left(D_{t}, p_{t}, p_{0 t}, \lambda_{2 t}, \tau, s\right), s \in[0, \infty)$. Apply 
Itô's lemma on both sides of Equation B.23, and we get

$$
F_{t} \sigma_{F, t}=\int_{0}^{\infty} H_{t}(s) \sigma_{H(s), t} d s .
$$

In addition, we have

$$
\begin{aligned}
\pi_{t} F_{t}-\pi_{t^{-}} F_{t^{-}} & =\pi_{t} \int_{0}^{\infty} H_{t}(s) d s-\pi_{t^{-}} \int_{0}^{\infty} H_{t^{-}}(s) d s \\
& =\int_{0}^{\infty}\left(\pi_{t} H_{t}(s)-\pi_{t^{-}} H_{t^{-}}(s)\right) d s .
\end{aligned}
$$

Taking conditional expectation of both sides with respect to $\nu$ yields

$$
\overline{\mathcal{J}}\left(\pi_{t} F_{t}\right)=\int_{0}^{\infty} \overline{\mathcal{J}}\left(\pi_{t} H_{t}(s)\right) d s .
$$

Finally, by Itô's Lemma, we can see

$$
F_{t} \mu_{F, t}=\int_{0}^{\infty} H_{t}(s) \mu_{H(s), t} d s-D_{t} .
$$

$D_{t}$ term shows up as $H\left(D_{t}, p_{t}, \lambda_{2 t}, \tau, 0 ; p_{0 t}\right)=D_{t}$. Then we have

$$
\begin{aligned}
& \mu_{F t^{-}}+\frac{D_{t^{-}}}{F_{t^{-}}}+\sigma_{\pi t^{-}} \sigma_{F t^{-}}^{\top}+\left(\bar{\lambda}_{1}\left(p_{t}\right)+\lambda_{2 t}\right) \frac{\overline{\mathcal{J}}\left(\pi_{t} F_{t}\right)}{\pi_{t^{-}} F_{t^{-}}} \\
= & \frac{1}{F_{t^{-}}}\left(\int_{0}^{\infty} H_{t^{-}}(s) \mu_{H(s), t^{-}} d s\right)+\frac{1}{F_{t^{-}}} \sigma_{\pi t^{-}}^{\top} \int_{0}^{\infty} H_{t^{-}}(s) \sigma_{H(s), t^{-}} d s \\
& \quad+\left(\bar{\lambda}_{1}\left(p_{t}\right)+\lambda_{2 t}\right) \frac{1}{\pi_{t^{-}} F_{t^{-}}} \int_{0}^{\infty} \overline{\mathcal{J}}\left(\pi_{t} H_{t}(s)\right) d s \\
= & \frac{1}{F_{t^{-}}} \int_{0}^{\infty} H_{t^{-}}(s)\left(\mu_{H(s), t^{-}}+\sigma_{\pi t^{-}} \sigma_{H(s), t^{-}}^{\top}+\left(\bar{\lambda}_{1}\left(p_{t}\right)+\lambda_{2 t}\right) \frac{1}{\pi_{t^{-}} H_{t^{-}}} \overline{\mathcal{J}}\left(\pi_{t} H_{t}(s)\right)\right) d s \\
= & \frac{1}{F_{t^{-}}} \int_{0}^{\infty} H_{t^{-}}(s)\left(-\mu_{\pi t^{-}}\right) d s \\
= & -\mu_{\pi t^{-}} \frac{1}{F_{t^{-}}} \int_{0}^{\infty} H_{t^{-}}(s) d s \\
= & -\mu_{\pi t^{-}},
\end{aligned}
$$

where $H_{t^{-}}(s)=H\left(D_{t^{-}}, p_{t^{-}}, \lambda_{2 t^{-}}, \tau^{-}, s ; p_{0 t^{-}}\right)$. 
Since $\mu_{\pi t}, \mu_{F t}, \frac{D_{t}}{F_{t}}, \sigma_{\pi t}$ and $\sigma_{F t}$ are continuous processes, we can get

$$
\mu_{\pi t}+\mu_{F t}+\frac{D_{t}}{F_{t}}+\sigma_{\pi t} \sigma_{F t}^{\top}+\left(\bar{\lambda}_{1}\left(p_{t}\right)+\lambda_{2 t}\right) \frac{\overline{\mathcal{J}}\left(\pi_{t} F_{t}\right)}{\pi_{t^{-}} F_{t^{-}}}=0 .
$$

During non-announcement periods, the expected return per unit of time $d t$ of asset $j$ is given by

$$
r_{t}^{j}=\mu_{F t}^{j}+\left(\bar{\lambda}_{1}\left(p_{t}\right)+\lambda_{2 t}\right) \frac{\overline{\mathcal{J}}\left(F_{t}^{j}\right)}{F_{t^{-}}^{j}}+\frac{D_{t}^{j}}{F_{t}^{j}} .
$$

The following lemma characterizes the instantaneous expected return of the claim of a stream of dividends.

Lemma B.3. For an asset with claim to a stream of dividend with time-t price $F^{j}\left(D_{t}, p_{t}, p_{0 t}, \lambda_{2 t}, \tau\right)$, its instantaneous premium at $t \in \mathcal{N}$ is given by

$$
r_{t}^{j}-r_{t}=-\sigma_{\pi t} \sigma_{F t}^{j \top}-\left(\bar{\lambda}_{1}\left(p_{t}\right)+\lambda_{2 t}\right) E_{\nu}\left[\frac{\pi_{t}-\pi_{t^{-}}}{\pi_{t^{-}}} \frac{F_{t}^{j}-F_{t^{-}}^{j}}{F_{t^{-}}^{j}}\right] .
$$

Proof. The expected instantaneous return of a dividend stream $F\left(D_{t}, p_{t}, \lambda_{2 t}, \tau ; p_{0 t}\right)$ is

$$
\begin{aligned}
r_{t}^{j} & =E_{t}\left[\frac{\left(d F_{t}^{j}+D_{t}^{j} d t\right) / F_{t}^{j}}{d t}\right] \\
& =\mu_{F t}^{j}+\frac{D_{t}^{j}}{F_{t}^{j}}+\left(\bar{\lambda}_{1}\left(p_{t}\right)+\lambda_{2 t}\right) \frac{\overline{\mathcal{J}}\left(F_{t}^{j}\right)}{F_{t^{-}}^{j}} \\
& =-\mu_{\pi t}-\sigma_{\pi t} \sigma_{F t}^{j \top}-\left(\bar{\lambda}_{1}\left(p_{t}\right)+\lambda_{2 t}\right) \frac{\overline{\mathcal{J}}\left(\pi_{t} F_{t}^{j}\right)}{\pi_{t^{-}} F_{t^{-}}^{j}}+\left(\bar{\lambda}_{1}\left(p_{t}\right)+\lambda_{2 t}\right) \frac{\overline{\mathcal{J}}\left(F_{t}^{j}\right)}{F_{t^{-}}^{j}} \\
& =r_{t}-\sigma_{\pi t} \sigma_{F t}^{j \top}+\left(\bar{\lambda}_{1}\left(p_{t}\right)+\lambda_{2 t}\right) \frac{\overline{\mathcal{J}}\left(\pi_{t}\right)}{\pi_{t^{-}}}-\left(\bar{\lambda}_{1}\left(p_{t}\right)+\lambda_{2 t}\right) \frac{\overline{\mathcal{J}}\left(\pi_{t} F_{t}^{j}\right)}{\pi_{t^{-}} F_{t^{-}}^{j}}+\left(\bar{\lambda}_{1}\left(p_{t}\right)+\lambda_{2 t}\right) \frac{\overline{\mathcal{J}}\left(F_{t}^{j}\right)}{F_{t^{-}}^{j}} \\
& =r_{t}-\sigma_{\pi t} \sigma_{F t}^{j \top}-\left(\bar{\lambda}_{1}\left(p_{t}\right)+\lambda_{2 t}\right) E_{\nu}\left[\frac{\pi_{t}-\pi_{t^{-}}}{\pi_{t^{-}}} \frac{F_{t}^{j}-F_{t^{-}}^{j}}{F_{t^{-}}^{j}}\right],
\end{aligned}
$$

where $r_{t}$ is the instantaneous riskfree rate. Subtract $r_{t}$ from both sides of Equation B.31, we obtain the instantaneous premium in Lemma B.3 
Proof of Theorem 5. From Equation 28 and A.19, we have

$$
E_{\nu}\left[\frac{\pi_{t}-\pi_{t^{-}}}{\pi_{t^{-}}} \frac{F_{t}-F_{t^{-}}}{F_{t^{-}}}\right]=E_{\nu}\left[\left(e^{-\gamma Z_{t}}-1\right)\left(e^{-\phi Z_{t}}-1\right)\right] .
$$

From Itô's Lemma, we know

$$
\sigma_{F t}=\left[\sigma, \frac{1}{F_{t}} \frac{\partial F_{t}}{\partial \lambda} \sigma_{\lambda} \sqrt{\lambda_{2 t}}\right] .
$$

Substituting two equations above in Lemma B.3, we then get the conclusion of Theorem 5 . 


\section{References}

Ai, H. and Bansal, R. (2017). Risk preferences and the macro announcement premium. forthcoming, Econometrica.

Barro, R. J. and Ursúa, J. F. (2008). Macroeconomic crises since 1870. Brookings Papers on Economic Activity, 39(1 (Spring)):255-350.

Benzoni, L., Collin-Dufresne, P., and Goldstein, R. S. (2011). Explaining asset pricing puzzles associated with the 1987 market crash. Journal of Financial Economics, 101(3):552 - 573 .

Cogley, T. and Sargent, T. J. (2008). Anticipated utility and rational expectations as approximations of bayesian decision making. International Economic Review, $49(1): 185-221$.

Duffie, D. and Epstein, L. G. (1992). Asset pricing with stochastic differential utility. Review of Financial Studies, 5(3):411-436.

Duffie, D. and Skiadas, C. (1994). Continuous-time asset pricing: A utility gradient approach. Journal of Mathematical Economics, 23:107-132.

Epstein, L. and Zin, S. (1989). Substitution, risk aversion and the temporal behavior of consumption and asset returns: A theoretical framework. Econometrica, 57:937-969.

Fama, E. F. (1970). Efficient capital markets: A review of theory and empirical work. Journal of Finance, 25:383-417.

La Porta, R., Lakonishok, J., Shleifer, A., and Vishny, R. (1997). Good news for value stocks: Further evidence on market efficiency. Journal of Finance, 52:858-874.

Lettau, M. and Wachter, J. A. (2007). Why is long-horizon equity less risky? a duration-based explanation of the value premium. Journal of Finance, 62:55-92.

Lintner, J. (1965). Security prices, risk and maximal gains from diversification. Journal of Finance, 20:587-615.

Liptser, R. S. and Shiryaev, A. N. (2001). Statistics of Random Processes: I, II. Springer-Verlag, New York, NY. 
Lucca, D. O. and Moench, E. (2015). The pre-FOMC announcement drift. Journal of Finance, 70(1):329-371.

Savor, P. and Wilson, M. (2013). How much do investors care about macroeconomic risk? evidence from scheduled economic announcements. Journal of Financial and Quantitative Analysis, 48(2):343-375.

Savor, P. and Wilson, M. (2014). Asset pricing: A tale of two days. Journal of Financial Economics, 113(2):171-201.

Sharpe, W. (1964). Capital asset prices: A theory of market equilibrium under conditions of risk. Journal of Finance, 19:425-444.

Stein, J. C. and Sunderam, A. (2017). Gradualism in monetary policy: A timeconsistency problem? forthcoming, Journal of Finance.

Tsai, J. and Wachter, J. A. (2015). Disaster risk and its implications for asset pricing. Annual Review of Financial Economics, 7(1):219-252.

Tsai, J. and Wachter, J. A. (2017). Pricing long-lived securities in dynamic endowment economies. Working paper, University of Oxford and University of Pennsylvania.

Wachter, J. A. (2013). Can time-varying risk of rare disasters explain aggregate stock market volatility? The Journal of Finance, 68(3):987-1035. 
Figure 1: Portfolio excess returns against CAPM betas



Notes: The figure shows average excess returns on announcement days (diamonds) and non-announcement days (squares) on beta-sorted portfolios in daily data from 1961.012016.09. On the horizontal axis is CAPM beta. Also shown are estimated regression lines for announcement day returns against beta (solid red) and non-announcement day returns against beta (dashed red). 
Figure 2: Portfolio excess returns against CAPM betas on announcement and nonannouncement days



Notes: The figure shows average excess returns on announcement days (diamonds) and non-announcement days (squares) on beta-sorted portfolios in daily data from 1961.01-2016.09 as a function of the CAPM beta. Also shown are estimated regression lines for announcement day returns against beta (solid red) and non-announcement day returns against beta (dashed red). We simulate 500 samples of artificial data from the model, each containing a cross-section of firms. The blue and grey dots show average announcement day and non-announcement day returns for each sample as a function of beta, respectively. 
Figure 3: Boxplots of simulated portfolio average excess returns on announcement and non-announcement days



Notes: We compute average excess returns on announcement and non-announcement days for a cross-section of assets in data simulated from the model. The red line shows the median for each portfolio across samples; the box corresponds to the interquartile range (IQR), and the whiskers correspond to the highest and lowest data value within $1.5 \times$ IQR of the highest and lowest quartile. We plot returns against the median CAPM beta across samples for each portfolio. The red solid and dashed lines are the empirical regression lines of portfolio mean excess returns against market beta on announcement and non-announcement days, respectively. 
Table 1: Summary statistics of the excess returns of 10 beta-sorted portfolios

\begin{tabular}{|c|c|c|c|c|c|c|c|c|c|}
\hline \multirow[b]{2}{*}{$k$} & \multicolumn{3}{|c|}{ Unconditional } & \multicolumn{3}{|c|}{ Announcement day } & \multicolumn{3}{|c|}{ Non-announcement day } \\
\hline & $E\left[R X^{k}\right]$ & $\sigma^{k}$ & $\beta^{k}$ & $E\left[R X^{k}\right]$ & $\sigma^{k}$ & $\beta^{k}$ & $E\left[R X^{k}\right]$ & $\sigma^{k}$ & $\beta^{k}$ \\
\hline 1 & 1.61 & 53.3 & 0.19 & 3.73 & 53.3 & 0.17 & 1.34 & 53.3 & 0.19 \\
\hline 2 & 1.91 & 59.4 & 0.44 & 7.28 & 59.2 & 0.42 & 1.22 & 59.4 & 0.44 \\
\hline 3 & 2.58 & 68.9 & 0.57 & 7.38 & 70.1 & 0.56 & 1.97 & 68.8 & 0.57 \\
\hline 4 & 2.69 & 77.6 & 0.68 & 7.88 & 77.1 & 0.65 & 2.03 & 77.6 & 0.68 \\
\hline 5 & 2.58 & 87.5 & 0.79 & 7.78 & 87.7 & 0.77 & 1.91 & 87.4 & 0.80 \\
\hline 6 & 2.61 & 95.5 & 0.89 & 8.51 & 96.0 & 0.86 & 1.85 & 95.5 & 0.89 \\
\hline 7 & 2.57 & 106.0 & 1.00 & 8.41 & 108.1 & 0.98 & 1.82 & 105.7 & 1.00 \\
\hline 8 & 2.38 & 118.0 & 1.12 & 10.50 & 120.4 & 1.10 & 1.34 & 117.6 & 1.12 \\
\hline 9 & 2.37 & 136.8 & 1.30 & 12.63 & 139.5 & 1.29 & 1.05 & 136.4 & 1.30 \\
\hline 10 & 2.35 & 176.6 & 1.65 & 17.94 & 177.7 & 1.62 & 0.35 & 176.3 & 1.66 \\
\hline
\end{tabular}

Note: Sample statistics for excess returns of ten beta-sorted portfolios. The sample period is 1961.01-2016.09. We show the sample mean excess returns $\left(E\left[R X^{k}\right]\right)$, standard deviation $\left(\sigma^{k}\right)$ and CAPM beta $\left(\beta^{k}\right)$. Each portfolio is labelled by $k$. Column 1-3 report estimates with all data available. Column 4-6 and column 7-9 use returns on announcement and nonannouncement days, respectively. The unit is bps per day. 
Table 2: Calibration and simulation parameters

\begin{tabular}{lc}
\hline \multicolumn{1}{c}{ Panel A: Basic parameters } \\
\hline Average log growth in consumption $\mu_{C}(\%)$ & 2.52 \\
Average log growth in dividend $\mu_{D}(\%)$ & 2.52 \\
Volatility of consumption growth $\sigma(\%)$ & 2 \\
Rate of time preference $\beta$ & 0.012 \\
Relative risk aversion $\gamma$ & 3 \\
Firm leverage $\phi$ & $U[1.5,7]$ \\
\hline \multicolumn{2}{c}{ Panel B: The process for $\lambda_{1 t}$} \\
\hline Probability of disaster in the good state $\lambda^{G}$ \\
Probability of disaster in the bad state $\lambda^{B}$ \\
Probability of switching to the bad state $\phi_{G B}$ \\
Probability of switching to good state $\phi_{B G}$ \\
$\quad$ Panel C: The process for $\lambda_{2 t}$ \\
\hline \multicolumn{2}{c}{0.054} \\
\hline Average probability of disaster $\bar{\lambda}_{2}$ \\
Mean reversion in disaster probability $\kappa$ \\
Volatility for disaster probability $\sigma_{\lambda}$ \\
\hline
\end{tabular}

Note: Parameter values for the main calibration, expressed in annual terms. 
Table 3: Distribution of simulated market betas of portfolios with different leverages on announcement and non-announcement days

\begin{tabular}{|c|c|c|c|c|c|c|}
\hline \multirow[b]{2}{*}{$k$} & \multicolumn{2}{|c|}{$\beta_{a}^{k}$} & \multicolumn{2}{|c|}{$\beta_{n}^{k}$} & \multicolumn{2}{|c|}{$\beta_{a}^{k}-\beta_{n}^{k}$} \\
\hline & Median & $90 \% \mathrm{CI}$ & Median & $90 \% \mathrm{CI}$ & Median & $90 \% \mathrm{CI}$ \\
\hline 1 & 0.46 & {$[0.38,0.54]$} & 0.46 & {$[0.38,0.54]$} & -0.00 & {$[-0.01,0.01]$} \\
\hline 2 & 0.63 & {$[0.54,0.71]$} & 0.63 & {$[0.55,0.71]$} & -0.00 & {$[-0.01,0.01]$} \\
\hline 3 & 0.75 & {$[0.66,0.82]$} & 0.75 & {$[0.67,0.82]$} & -0.00 & {$[-0.01,0.01]$} \\
\hline 4 & 0.84 & {$[0.77,0.91]$} & 0.84 & {$[0.77,0.91]$} & -0.00 & {$[-0.01,0.01]$} \\
\hline 5 & 0.92 & {$[0.86,0.98]$} & 0.92 & {$[0.86,0.98]$} & -0.00 & {$[-0.01,0.01]$} \\
\hline 6 & 1.00 & {$[0.94,1.05]$} & 1.00 & {$[0.94,1.05]$} & 0.00 & {$[-0.00,0.00]$} \\
\hline 7 & 1.07 & {$[1.02,1.12]$} & 1.07 & {$[1.02,1.12]$} & 0.00 & {$[-0.00,0.00]$} \\
\hline 8 & 1.13 & {$[1.08,1.20]$} & 1.13 & {$[1.08,1.20]$} & 0.00 & {$[-0.00,0.00]$} \\
\hline 9 & 1.20 & {$[1.14,1.28]$} & 1.20 & {$[1.14,1.28]$} & 0.00 & {$[-0.01,0.01]$} \\
\hline 10 & 1.27 & {$[1.20,1.36]$} & 1.27 & {$[1.21,1.36]$} & 0.00 & {$[-0.01,0.01]$} \\
\hline 11 & 1.34 & {$[1.27,1.45]$} & 1.34 & {$[1.27,1.44]$} & 0.00 & {$[-0.01,0.01]$} \\
\hline 12 & 1.42 & {$[1.34,1.54]$} & 1.42 & {$[1.34,1.54]$} & 0.00 & {$[-0.01,0.01]$} \\
\hline
\end{tabular}

Note: For each sample, portfolio market betas $(\beta)$ on announcement (footnote $a$ ) and nonannouncement (footnote $n$ ) days are computed, respectively. The market is defined as the weighted average of portfolios. This table reports summary statistics of the distribution across samples. 
Table 4: Empirical values and simulated distributions of regression slope coefficient of excess returns on portfolio betas on announcement and non-announcement days.

\begin{tabular}{lrrr}
\hline Coefficient & Data & Simulation Median & $90 \%$ CI \\
\hline$\delta_{a}$ & 10.47 & 10.52 & {$[8.19,13.66]$} \\
$\delta_{n}$ & 1.28 & 2.24 & {$[1.25,4.07]$} \\
$\delta_{a}-\delta_{n}$ & 9.19 & 8.31 & {$[5.65,11.31]$} \\
\hline
\end{tabular}

Note: For each sample, the regression $E_{i}\left[R X_{t}^{k}\right]=\delta_{i} \beta_{i}^{k}+\eta_{i}^{k}$ is estimated, where $i=a, n$ stands for sets of accouncement and non-announcement days, respectively. $k$ stands for different beta-sorted portfolios. The first column reports the regression coefficients in empirical analysis, using beta-sorted portfolios. The $90 \%$ confidence intervals are computed using simulation samples. 
Table 5: The equity premium and volatility on announcement and nonannouncement days

\begin{tabular}{lrrr}
\hline Statistic & Data & Simulation Median & $90 \%$ CI \\
\hline$E_{a}\left[R X_{t}^{\mathrm{mkt}}\right]$ & 10.66 & 10.40 & {$[8.08,13.54]$} \\
$\operatorname{std}_{a}\left[R X_{t}^{\mathrm{mkt}}\right]$ & 102.2 & 55.4 & {$[39.8,72.7]$} \\
$E_{n}\left[R X_{t}^{\mathrm{mkt}}\right]$ & & 2.24 & {$[1.26,4.07]$} \\
$\operatorname{std}_{n}\left[R X_{t}^{\mathrm{mkt}}\right]$ & 1.27 & 55.4 & {$[39.7,72.3]$} \\
$E_{a}\left[R X_{t}^{\mathrm{mkt}}\right]-E_{n}\left[R X_{t}^{\mathrm{mkt}}\right]$ & 98.3 & 8.19 & {$[5.53,11.21]$} \\
$\operatorname{std}_{a}\left[R X_{t}^{\mathrm{mkt}}\right]-\operatorname{std}_{n}\left[R X_{t}^{\mathrm{mkt}}\right]$ & 3.9 & -0.0 & {$[-2.5,2.5]$} \\
\hline
\end{tabular}

Notes: $E_{a}\left[R X_{t}^{\mathrm{mkt}}\right]$ and $E_{n}\left[R X_{t}^{\mathrm{mkt}}\right]$ denote the average excess return on the market portfolio on announcement days and non-announcement days respectively. $\operatorname{std}_{a}\left[R X_{t}^{\mathrm{mkt}}\right]$ and $\operatorname{std}_{n}\left[R X_{t}^{\mathrm{mkt}}\right]$ denote analogous statistics for the standard deviation. The first column reports the empirical estimate. The second column reports the median across samples simulated from the model. The third column reports the two-sided 90th percentile confidence interval from simulated samples. 